\title{
eNeuro
}

Research Article: New Research / Cognition and Behavior

\section{Brain Dynamics of Action Monitoring in Higher- Order Motor Control Disorders: The Case of Apraxia}

https://doi.org/10.1523/ENEURO.0334-20.2021

Cite as: eNeuro 2022; 10.1523/ENEURO.0334-20.2021

Received: 3 August 2020

Revised: 5 December 2021

Accepted: 15 December 2021

This Early Release article has been peer-reviewed and accepted, but has not been through the composition and copyediting processes. The final version may differ slightly in style or formatting and will contain links to any extended data.

Alerts: Sign up at www.eneuro.org/alerts to receive customized email alerts when the fully formatted version of this article is published.

Copyright (? 2022 Spinelli et al.

This is an open-access article distributed under the terms of the Creative Commons Attribution 4.0 International license, which permits unrestricted use, distribution and reproduction in any medium provided that the original work is properly attributed. 
1 Title: Brain dynamics of action monitoring in higher-order motor control disorders: the case 2 of apraxia

3 Abbreviated title: Altered EEG markers of error-monitoring in limb apraxia

5 Authors: Giuseppe Spinelli ${ }^{1,2 *}$, Rachele Pezzetta ${ }^{1,3 *}$, Loredana Canzano ${ }^{1}$, Emmanuele

6 Tidoni $^{1,4} \&$ Salvatore Maria Aglioti ${ }^{1,5}$

$8{ }^{1}$ IRCCS Santa Lucia Foundation, via Ardeatina 306, 00179 Rome, Italy;

$9{ }^{2}$ myBrainTechnologies, 85 rue de Maubeuge, 75010, Paris, France;

$10{ }^{3}$ IRCCS San Camillo Hospital,Venice, Italy;

$11{ }^{4}$ Department of Psychology, University of Hull, UK;

$12{ }^{5}$ Sapienza Università di Roma e Istituto Italiano di Tecnologia, Italy

* these authors contributed equally to the work

Authors' contribution: GS and SMA conceived the study. GS, LC and RP performed the

experiment and collected the data. GS analysed EEG data. LC analysed neuropsychological and neuroimaging data. ET designed the stimuli and programmed the set-up. All the authors discussed the protocol and the results and contributed to manuscript preparation.

Correspondence should be addressed to: Salvatore Maria Aglioti

(salvatoremaria.aglioti@uniroma1.it) or Giuseppe Spinelli (giuseppespinelli88@gmail.com)

Acknowledgments: The authors are grateful to all the patients and their relatives.

Number of pages: 36; Number of figures: 6; Number of tables: 6

Words-Abstract: 240; Words-Significance Statement: 109; Words-Introduction: 739;

Words-Discussion: 2022

Conflict of interest: The authors declare no conflict of interest

Funding: The study was supported by PRIN grant (Progetti di Ricerca di Rilevante Interesse 


\section{Abstract}

36 Limb Apraxia (LA) refers to a high-order motor disorder characterized by the inability to

37 reproduce transitive actions upon commands or after observation. Studies demonstrate that

38 action observation and action execution activate the same networks in the human brain, and

39 provides an onlooker's motor system with appropriate cognitive, motor and sensory-motor

40 cues to flexibly implementing action-sequences and gestures. Tellingly, the temporal

41 dynamics of action monitoring has never been explored in people suffering from LA. To fill

42 this gap, we studied the electro-cortical signatures of error observation in human participants

43 suffering from acquired left-brain lesions with (LA+) and without (LA-) limb apraxia, and in

44 a group of healthy controls $(\mathrm{H})$. EEG was acquired while participants observed from a first-

45 person perspective an avatar performing correct or incorrect reach-to-grasp a glass action in

46 an immersive-virtual environment. Alterations of typical EEG signatures of error observation

47 in time (early error positivity) and time-frequency domain (theta band-power) were found

48 reduced in $\mathrm{LA}+$ compared to $\mathrm{H}$. Connectivity analyses showed that $\mathrm{LA}+$ exhibited a

49 decreased theta phase synchronization of both the fronto-parietal and fronto-frontal network,

50 compared to $\mathrm{H}$ and LA-. Moreover, linear regression analysis revealed that the severity of

51 limb apraxia (TULIA scores) was predicted by mid-frontal error-related theta activity,

52 suggesting a link between error monitoring capacity and apraxic phenotypes. These results

53 provide novel neurophysiological evidence of altered neurophysiological dynamics of action

54 monitoring in individuals with LA and shed light on the performance monitoring changes

55 occurring in this disorder. 


\section{SIGNIFICANCE STATEMENT}

57

Combining EEG and immersive virtual reality we provide novel neurophysiological evidence of altered performance monitoring in apraxic patients. We show that the observation of incorrect actions performed by an avatar seen from a first-person perspective elicited reduced electrocortical markers of error detection in apraxic patients. Tellingly, apraxia severity predicted reduction of mid-frontal theta activity, regardless of brain lesion volume and patients' cognitive capacity. The results shed new light on the possible neurophysiological signatures of the link between limb apraxia and performance monitoring. Moreover, our EEG-virtual reality paradigm may provide a new tool for investigating the brain dynamics of monitoring action errors also in brain damaged patients with motor limitations.

Legend: Legend: LA+: acquired left-brain lesions with limb apraxia; LA-: acquired left-brain lesions without limb apraxia; TULIA: test of upper limb apraxia; CAVE: cave automatic virtual environment; VAS: visual analog scale; Ow: feeling of ownership; Ag: feeling of agency; oERN: observation error-related negativity; oPe: observed error positivity.

3

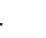

(1)

6

7

8


85

86

87

88

89

90

91

92

93

\section{Introduction}

Limb Apraxia (LA) is a disorder of higher order motor control mainly associated with damage of left fronto-parietal brain networks (Buxbaum et al., 2014; 2018). LA is characterized by a complex combination of perceptual (Halsband et al., 2001), motor (Candidi et al., 2017), and cognitive (Rothi et al., 1985) deficits whose interaction ultimately affects the implementation of transitive and intransitive movements upon verbal command or imitation. According to the 'affordance competition hypothesis' (Cisek, 2007), potential actions compete against each other, and information is collected to bias and solve this competition until a response is selected. Competition arises from mere sensory exposition to an object and its physical properties that automatically triggers conflicting action schema for 'affording' the object itself (Cisek, 2007; Cisek and Kalaska, 2010), and may lead to performance errors if the conflict is not resolved (Cooper, 2007). Tellingly, apraxic patients not only display deficits in action execution but also in action understanding and simulation (Cubelli et al., 2000; Rothi et al., 1985), in mental action imagery task (Sirigu et al., 1999), in generating internal models for action execution (Buxbaum et al., 2005), and in the judgment of the correctness of seen or heard (Pazzaglia et al., 2008a; Aglioti and Pazzaglia, 2010; 2011; Canzano et al., 2014) actions. Moreover, deficits in action monitoring were positively correlated with difficulties in action execution (Pazzaglia et al., 2008a), thus corroborating the hypothesis of a direct matching between action perception and execution. In line with the affordance competition hypothesis, studies suggest that errors in apraxia could be due to a deficient resolution of competition between action selection (Jax \& Buxbaum, 2013; Buxbaum et al., 2014; Watson \& Buxbaum, 2015) or to a failure to resolve affordance competition (Rounis \& Humphreys, 2015). In keeping with Bekkering and colleagues (2000), when an action is observed, it is the action goal that is observed, and not just a movement. Action observation and execution are bidirectionally linked, so that motor skills may improve 
as an effect of merely seeing others moving (Ertel et al., 2007; Cross et al., 2008; Ernst and

111 Steinhauser, 2017). Moreover, to perform specific actions improves the ability to perceive

112 them (Casile and Giese, 2004; Lepage and Theoret, 2006). Monitoring actions through

113 observation implies the evaluation of their correctness. EEG studies demonstrate that

114 observation of errors in one's own and others' actions elicits specific markers over the mid-

115 frontal cortex, namely: i) the observer Error-Related Negativity (oERN), the observer error

116 Positivity (oPe; van Schie et al., 2004; de Brujin et al., 2007), and ii) increased power in the

117 theta band (4-8 Hz; Cavanagh et al., 2009; 2010; 2012). These patterns of electro-cortical

118 brain activity are likely associated to conflict processing and resolution (Cavanagh and Frank,

119 2014). Conflict arises when a unique (correct) action should be selected among a set of 120 competing (incorrect) actions and serves as an alarm signal conveyed from the mid-frontal to 121 the lateral pre-frontal and posterior brain areas to increase cognitive control over actions 122 (Cohen and Cavanagh, 2011; van Driel et al., 2012).

The present study aims to investigate the temporal dynamics of action monitoring in patients suffering from LA by linking the 'affordance competition theory' and the 'conflict monitoring model'. Crucially, both theories consider conflict processing as a fundamental mechanism by which the performance monitoring system exerts motor and cognitive control over actions. In view of the affordance-competition hypothesis, we predict that patients with LA tend to experience high levels of conflict during goal-directed action monitoring, which arises from the competition between correct and incorrect action schemas. This may lead to an exaggerated burden of unresolved conflict that impairs the operation of the action monitoring

131 system. Capitalizing on previous similar reports (Spinelli et al., 2018; Pezzetta et al, 2018; 132 Pavone et al., 2016), we recorded EEG in left-brain damaged individuals with and without 133 limb apraxia and in a control group who observed through immersive virtual-reality an avatar 134 performing correct or incorrect actions. In line with previous studies on error monitoring, 
awareness, and gesture recognition in patients with apraxia (Canzano et al., 2014; Canzano et al., 2016; Candidi et al., 2016; Scandola et al., 2021), we expected an impairment in patients with LA when the error monitoring system is called into play, that is when a mismatch between predicted and observed action goal occurs. Acquiring EEG signatures of performance monitoring during the observation of correct and incorrect actions provided novel information upon the integrity of the error detection system in LA.

\section{Methods}

\section{Participants}

Twelve right-handed, left-brain damaged patients were recruited from the local NeuroRehabilitation Unit between March and August 2016. They had suffered from focal vascular lesions (e.g. patients with traumatic brain injuries were not included) between 292 and 1095 days (LA+: $\mathrm{M}=580.33$; $\mathrm{SD}=252.48$; $\mathrm{LA}-\mathrm{M}=687.17, \mathrm{SD}=207.08$ ), thus they were tested during chronic phase. A primary inclusion criterion was the ability to perform the task (EEGVR session), and to understand the task instructions. All the participants signed an informed consent for participation. Based on a neuropsychological assessment (Table 1) of their symptoms, participants were divided in two groups: i) patients with (LA+; $n=6,4$ males, 2 females) and ii) without (LA-; n= 6, 3 males, 3 females) Limb Apraxia. The two groups were matched for age (mean age $\pm \mathrm{SD}$ : $\mathrm{LA}^{+}=63.1 \pm 14.4$ years, $\mathrm{LA}-=58.5 \pm 14.2$ years) and education (LA-: $12 \pm 2.0$; LA+: $13.8 \pm 3.4$ ). An age-and-gender matched (mean age $\pm \mathrm{SD}$ : $62.4 \pm 11.2,6$ males, 4 females) sample of 10 healthy participants $(\mathrm{H})$ was also tested. An age-and-gender matched (mean age \pm SD: $62.4 \pm 11.2$ ) sample of 10 healthy participants $(H)$ was also tested. The study was conducted in accordance with the guidelines of Declaration of Helsinki and approved by the local Ethics Committee. 
In order to inform on the patients' cognitive profile, standard tests and batteries for

160 general neuropsychological assessment were administered (for details, see Table 1),

161 including: general cognitive abilities (Raven, Court, \& Raven, 1988), executive functions

162 (non-verbal subtests of the Frontal Assessment Battery - Appollonio et al., 2005) and spatial

163 attention (Line Bisection; Wilson, Cockburn, \& Halligan, 1987). Verbal comprehension and

164 denomination subtests of the Aachener Aphasia Test (Luzzatti, Willmes, \& De Bleser, 1996)

165 were used to assess language comprehension deficits. Given that the experimental task

166 implied the mere observation of correct vs erroneous upper limb actions, the assessment of

167 apraxia focused on tests where actions implied the use of upper limbs, namely the ideomotor

168 (TULIA; Vanbellingen et al., 2010), and the Ideational apraxia tests (De Renzi and Lucchelli,

169 1988). The two groups did not differ in ideational apraxia (see Table 1) suggesting that

170 semantic knowledge concerning actions was preserved. While LA+ presented difficulty in

171 understanding words with respect to LA-, no such effect was found for sentence

172 comprehension. This result, together with the nature of the task, suggests that comprehension

173 did not play a major role in the experimental effects.

174

175

176

177

178

179

180

181

182

183

Analysis of brain lesions was carried out for LA- and LA+ by means of the MRIcron software (https://www.nitrc.org/projects/mricron; Rorden \& Brett, 2007; 2011). The MRI/CT scans available for all the patients were mapped by drawing on the standard T1-weighted MRI template (ICBM152) of the Montreal Neurological Institute (MNI) coordinate system, approximately oriented to match the Talairach space (Talairach \& Tournoux, 1988). The standard template (size: $181 \times 217 \times 181 \mathrm{~mm}$, voxel resolution: $1 \mathrm{~mm}^{2}$ ) was rotated on the three planes in order to match each patient's MRI/CT scan orientation as closely as possible. Then, two experienced clinicians (who were blind as to which patients the scan belonged to) traced any lesion manually on the axial slices of the rotated template, while another one checked all the drawings in a double-blind procedure. For each patient the outcome was a map of the 
damaged areas with each voxel labelled as 0 (intact) or 1 (lesioned). All the lesion maps were rotated back to the canonical orientation in order to align them to the standard stereotaxic

186 MNI space (in $2 \mathrm{~mm} \times 2 \mathrm{~mm} \times 2 \mathrm{~mm}$ voxel). After that, maps were filtered with a custom mask based on the ICBM152 template to exclude the voxels of lesions outside the white and grey matter brain tissues. Each patient's lesion was superimposed onto T1 templates to calculate the number of lesioned voxels in various cerebral areas, and the center of the mass of each damaged area. This was then overlapped onto the Automatic Anatomical Labelling (AAL) template (Tzourio-Mazoyer et al., 2002) to provide information on the grey matter, and onto the JHU white-matter atlas (Dr. Susumu Mori, Laboratory of Brain Anatomical MRI, Johns Hopkins University) for the white matter. LA+ and LA- lesion overlap and lesion subtraction were performed to highlight patients' lesional patterns (Figure 1). For each region, the MNI coordinates of the center of mass along with the number (n) and percentage (\%) of clustering voxels are provided for LA+ LA- and subtraction lesion map (Table 4 and Table 5). Analysis of tract disconnection probability were also carried out, by mapping the lesion from each patient onto tractography reconstructions of white matter pathways obtained from a group of healthy controls (Rojkova et al., BSF 2015). We quantified the severity of the disconnection by measuring the probability of specific tracts (Thiebaut de Schotten et al. 2014) using Tractotron software as part of the BCBtoolkit (Foulon et al. 2018; http://www.toolkit.bcblab.com; Table 6). We computed t-test comparison with falsediscovery rate correction to verify significant differences between groups.

\section{Apparatus and virtual environment}

Participants were seated in a four screens $(3 \times 3 \times 2.5 \mathrm{~m})$ CAVE system (Cruz-Neira et rate of $60 \mathrm{~Hz}$ by Nvidia Stereo Glasses, which were in turn interfaced with an Intersense 900 
ultrasonic system (Thales Visionix; 6 degrees of freedom). The virtual scenario included a

210 virtual room $(3 \times 3 \times 2 \mathrm{~m})$ with a virtual table, and an avatar with both its right $(\mathrm{R})$ and left

211 (L) upper limb on the table (Figure 2 panel B). Atop the table was a yellow support with the

212 virtual glass placed on it. The virtual scenario and the avatar were drawn on a 1:1 scale by

213 Maya 2011 and 3ds Max 2011 (Autodesk, Inc) respectively, and rendered by XVR 2.1

214 (Huang et al., 2013; Tecchia et al., 2010). The avatar's kinematics were implemented using

215 Halca libraries (Gillies and Spanlang, 2010). Marker events were sent to the EEG by means of

216 a custom-made circuit governed by a digital input/output device (PoKeys 55; PoLabs;

\section{7 https://www.poscope.com).}

\section{Experimental design}

Expanding on previous reports (Pavone et al., 2016; Spinelli et al., 2018; Pezzetta et al., 2018), the main task used in this study implied that participants observed correct or incorrect reach-to-grasp a glass actions performed by an avatar seen from a first-person perspective (1PP) . Participants were immersed in the virtual scenario and their physical body was aligned with the virtual body in order to maximize embodiment. The participants' real body was occluded by a black cloth. Each trial started with an Inter Trial Interval (ITI) lasting $1250 \mathrm{~ms}( \pm 250 \mathrm{~ms})$ in which both avatar's upper limbs rested on the table. After a synthesized voice instructed the avatar to grasp the glass $(2000 \mathrm{~ms})$, participants observed one of the two avatar's limbs ( $\mathrm{R}$ or $\mathrm{L}$, depending on the experimental block) reaching and grasping the virtual glass (Figure 1 panel B). Each reach-to-grasp action lasted $1000 \mathrm{~ms}$, such 230 that the first $700 \mathrm{~ms}$ were identical for all actions, and the last $300 \mathrm{~ms}$ defined a correct (C) or 231 incorrect (I) outcome. While correct actions resulted in a successful grasping of the virtual 232 glass, incorrect actions depicted a virtual limb directed 5-virtual-cm right-ward the virtual 233 glass (or 5 virtual-cm left-ward in the case of left arm movements). Two-thousand 
milliseconds (2000 ms) elapsed after the completion of each action, before the virtual limb

235 returned to its starting position. The whole experiment counted 120 trials, split in two blocks

236 of 60 trials, each containing R or L avatar's actions exclusively. The order of blocks ( $\mathrm{R}$ and

237 L) was counter-balanced within participants for each group (LA+, LA- and H). Correct ( $\mathrm{n}=$

238 36) and Incorrect $(\mathrm{n}=24)$ actions were randomly presented across the trial-list of each block.

Subjective ratings of virtual embodiment (i.e., sense of ownership and vicarious agency) were collected in the $25 \%$ of trials (i.e., 30 trials). Participants were asked to separately rate on two Visual-Analogue Scale (VAS) i) how strongly the virtual arm was felt as part of their body (feeling of Ownership; Ow), and ii) how much they felt in control of the virtual arm (feeling of Vicarious Agency; Ag). Ratings were acquired at the end of avatar's actions, by asking participants to quantify the strength of their feelings by positioning a virtual stick on the VAS ranging from 0 to 100 , where 0 indicated 'no feeling' and 100 'highest feeling'. The different VASs were sequentially displayed on a black box appearing ahead the virtual glass and disappearing immediately after an answer was provided. Each participant provided a total of 15 self-reports of $\mathrm{Ow}$ and $\mathrm{Ag}$ in each block, 9 for $\mathrm{C}$ and 6 for I. The order of Ow and Ag self-reports was counter-balanced across trials.

\section{EEG recording and analysis}

EEG data were acquired by means of tin electrodes embedded in a fabric cup (Electro-

Cap International, Inc.), according to the 10-10 system, from 60 scalp sites (Spinelli et al., 2018). The electrode on the right earlobe served as online reference, while the ground electrode was placed on AFz. A bipolar electro-oculogram was recorded from two electrodes placed on the lateral end of the bicanthal plane. The signal was recorded by a Neuroscan SynAmpsRT (Compumedics, ltd) at $1000 \mathrm{~Hz}$, and filtered with a hardware band-pass of 0.05$200 \mathrm{~Hz}$. All impedances were kept below $5 \mathrm{~K} \Omega$. EEG traces were processed using the 
FieldTrip toolbox (Oostenveld et al., 2011; release: 20170607) in Matlab R2016a (The

260 MathWorks, Inc.).

For each subject, continuous EEG signals were filtered offline with a $0.5 \mathrm{~Hz}$ high-pass

262

263

264 FIR filter (onepass, zero-phase) and locked to the onset of the avatar's arm-path deviation (i.e., $300 \mathrm{~ms}$ before action-offset). This time-point corresponded to the latest timeframe in which observed grasping trajectories were still identical between correct and incorrect actions (Spinelli et al., 2018). Epochs of $6 \mathrm{~s}( \pm 3 \mathrm{~s}$ around this trigger) were extracted and sorted according to the ACCURACY of the observed avatar's action (2 levels: correct $[\mathrm{C}]$ and incorrect $[\mathrm{I}]$ ), and to the avatar's LIMB that was observed (2 levels: right [R] and left [L]). Blinks and oculomotor artifacts were removed by the Independent Components Analysis (ICA). On average, 3.6 components (range: 1-7) referring to blink/oculomotor artifacts were discarded. Trials exhibiting residual artifacts were discarded by means of i) a summary plot of 3 metrics (variance, z-score, kurtosis) of all channels, as implemented in FieldTrip, and ii) a further visual inspection of all segments and all channels. Details of remaining trials are shown in Table 2. The obtained artifact-free time series were then re-referenced to the common-average reference and baseline corrected with respect to a time window of $200 \mathrm{~ms}$ prior to the trigger (i.e., the onset of avatar's arm-path deviation). Time- (ERPs), timefrequency (TF) domain and phase connectivity analyses were carried out.

For ERPs analysis, the across-trials average for each condition (LIMB [R, L] * ACCURACY [C, I]) was obtained in the time-range of -200 to $800 \mathrm{~ms}$. This time-window was considered for statistical analyses. TF analysis was carried out by means of the wavelets method. Width (or cycles) of each wavelet was 4 (i.e., $4 / 2 \pi f$ ). Frequency resolution was $1 \mathrm{~Hz}$ (range: 4-30 Hz). Length of the time window for computation was $2.6 \mathrm{~s}( \pm 1.3 \mathrm{~s}$ around the trigger). Time-resolution was $50 \mathrm{~ms}$. TF spectra were corrected to the relative signal change ( $\%$ change) of the event period (from 0 to $1000 \mathrm{~ms}$ ) with respect to the baseline (from -200 to 
0). The average across trails for each condition was calculated in the time-window from -200

285 to 1000 . This time-window was used for statistical analyses. Functional connectivity analysis

286 was carried out by computing the trial-by-trial phase locking value (PLV; Lachaux et al., 287 1999) for across channels combinations. Imaginary coherence was considered to compensate 288 for volume conduction issues (Vinck et al., 2011). Oscillatory phase synchronization between 289 channels is considered a connectivity measure that reflects the exchange of information between neuronal populations (Sauseng and Klimesch, 2008).

\section{Statistical Analysis}

In order to statistically estimate time- and time-frequency differences between groups 294 (LA+ vs. LA- vs. H) and within conditions (LIMB and ACCURACY) at each electrode, a non-parametric Monte Carlo permutation was carried out (1000 repetitions). As first, a permutation distribution of the significance probabilities for dependent-samples t-tests between R vs. L was calculated separately for each group. Since no significant results were obtained (all $\mathrm{p}>.05$ ), voltage/power values of both conditions ( $\mathrm{R}$ and $\mathrm{L}$ ) were averaged. On these obtained time-series, dependent-samples t-tests were carried out to estimate the differences between C vs. I separately for each group using non-parametric cluster-based permutation analysis as implemented in Fieldtrip (cluster-alpha $=.05$ ). Contrasts between groups were computed by means of three independent-samples t-tests ( $\mathrm{H}$ vs. LA+, H vs. LA-, LA- vs. LA+) using voltage/power values difference between incorrect and correct conditions (I minus C). To correct for multiple comparisons, a cluster-based correction was applied to all tests as implemented in FieldTrip (cluster-alpha $=.05$; Maris and Oostenveld, 2007). averaged as no difference was found $(\mathrm{p}>.05)$. Transient theta phase activity from mid-frontal to lateral pre-frontal and parieto-occipital brain areas have been shown to reflect a functional 
mechanism to increase post-error cognitive control and sensory attention (Cohen et al., 2009;

310 Cavanagh et al., 2009; Cohen and Cavanagh, 2011) respectively. Thus, PLVs were calculated

311 for all channel-combination and all frequencies in the time-window from -200 and $1000 \mathrm{~ms}$.

312 Then, connectivity measure between mid-frontal (electrodes FC1, FCz, FC2, C1, Cz, C2),

313 lateral pre-frontal (electrodes F3, F5, F4, F6) and parieto-occipital (electrodes PO7, PO3,

$314 \mathrm{POz}, \mathrm{PO} 4, \mathrm{PO} 8, \mathrm{O} 1, \mathrm{Oz}$, and $\mathrm{O} 2$ ) scalp regions were extracted for each participant in three

315 separate time windows, i.e. $200-400,400-600$, and 600-800 ms. Dependent-samples t-tests

316 were carried out to test any difference between conditions (C vs. I). Differences between

317 groups (LA+ vs. LA- vs. H) were estimated by means of a between-subject ANOVA, using

318 groups (LA+ vs. LA- vs. H) as main factor and the differences between incorrect and correct

319 condition (I minus C) as dependent measures.

Finally, the relation between signs and symptoms of limb apraxia and brain markers of error monitoring was investigated by means of a multiple linear regression model predicting error-related band power changes from LA phenotypes (LA+, LA-), TULIA scores (normalized in z-scores), total brain lesioned volume ( $\mathrm{c}^{3}$ normalized in $\mathrm{z}$-scores) and the FAB scores (normalized in z-scores); i.e. $Y_{i}=\beta_{0}+\beta_{i} X_{i+\text { interactions terms }} \varepsilon_{\mathrm{I}}$. Data of all the patients (LA+ and LA-) were included in the linear model, thus allowing to test which of the main predictors or their interaction terms, predicted error-related EEG dynamics. The brain lesioned volume and the FAB scores were chosen in the regression model in order to control for two clinically relevant indices that could account for by the variance between the three groups of patients, namely any structural difference between patients' brain and any difference in executive abilities. In keeping with the time-frequency analyses, power spectra in $\mathrm{R}$ and $\mathrm{L}$ condition were averaged, and the difference between incorrect minus correct condition was obtained. From these obtained values, beta coefficients for the main effects and 
the interactions terms, and their p-values were calculated for each electrode and each time

334 (from 500 to $1000 \mathrm{~ms}$ ) - frequency (from 4 to $30 \mathrm{~Hz}$ ) point across the whole patients' sample.

\section{Results}

\section{Time-domain analysis}

Permutation tests resulting from the contrast between incorrect vs. correct conditions revealed significant positive clusters only for $\mathrm{H}$ (Figure 3 panel A). In particular, a significant voltage increase was found in incorrect trials in the 430 to $550 \mathrm{~ms}$ time-window, at a midfrontal (t-max: 2.74, $\mathrm{p}<.001$, electrode FCz; Figure 3 panel B) and occipital (t-max: 3.27, p < .001 , electrode $\mathrm{Oz}$ ) cluster. No negative cluster was found from this analysis. The independent-samples t-tests carried out between groups (LA+ vs. LA-, LA+ vs. H, LA- vs. H;

Figure 3 panel C) revealed positive clusters only for the contrast between $\mathrm{H}$ and $\mathrm{LA}+$. In this, $\mathrm{H}$ exhibited increased voltage in the time window from 420 to $560 \mathrm{~ms}$ at mid-frontal (t-max: 2.36, $\mathrm{p}<.001$; electrode FC3) and parieto-occipital (t-max: 3.01, $\mathrm{p}<.001$, electrode $\mathrm{Oz}$ ) 347 clusters.

\section{Time-frequency domain analysis}

As for ERPs, the contrast between incorrect vs. correct conditions revealed significant clusters only for the $\mathrm{H}$ group. More specifically, a significant increase of theta-band (4-8 Hz) was found in incorrect trials in the time range running from 300 to $650 \mathrm{~ms}$ at a mid-frontal cluster (t-max: 4.78, $\mathrm{p}<.001$, electrode FCz; Figure 4 panel A). The independent-samples ttests between groups (LA+ vs. LA- vs. H; Figure 4 panel B) revealed positive clusters only for the contrast $\mathrm{H}$ vs $\mathrm{LA}+$, accounted for by the fact that $\mathrm{H}$ exhibited increased theta power in the time range 420-575 ms at mid-frontal (t-max: 2.39, $\mathrm{p}<.001$; electrode $\mathrm{FC1}$ ) and parietooccipital (t-max: 2.74, $\mathrm{p}<.001$, electrode CP1) clusters. 


\section{Connectivity analysis}

Mid- to lateral- frontal connectivity. The dependent-samples t-tests carried out

359 between incorrect vs. correct condition revealed significant effects only for $\mathrm{H}(\mathrm{t}=2.18, \mathrm{p}<$

$360.016)$ in the time window from 400 to $600 \mathrm{~ms}$. The effect was explained by an increased theta

361 phase connectivity for the observation of incorrect actions (Figure 5 left panel). No further significant effect was found in any other time-windows. The significant effect of the betweensubjects ANOVA $\left(\mathrm{F}_{2,43}=5.43, \mathrm{p}<.01\right)$ was explained by a lower theta phase connectivity in $\mathrm{LA}+$ (mean: -.02, range: $-.01-.05)$ with respect to both LA- (mean: .04, range: $-.1-.16)(\mathrm{p}<$

$.05)$ and $\mathrm{H}$ (mean: .05, range: $-.05-.26 ; \mathrm{p}<.001)$ in the same time-range (i.e., 400-600 ms).

No further effect was found.

Mid-frontal to parieto-occipital connectivity. The dependent-samples t-tests computed between incorrect vs. correct condition revealed multiple significant effects. An increased error-related theta phase synchronization was found for both LA- $(\mathrm{t}=2.53, \mathrm{p}<.02)$ and $\mathrm{H}(\mathrm{t}=$ $2.68, \mathrm{p}<.01)$ in the time-window from 200 to $400 \mathrm{~ms}$. This effect remained significant also in the subsequent time window (i.e., 400-600) only for $\mathrm{H}(\mathrm{t}=2.64, \mathrm{p}<.02)$. No significant effect was found in the time-window from 600 to $800 \mathrm{~ms}$. The significant effect of the between-subjects ANOVA $\left(\mathrm{F}_{2,43}=3.91, \mathrm{p}<.02\right)$ was explained by a decreased theta phase connectivity in LA+ (mean: -.01, range: $-.01-.03$ ) with respect to both LA- (mean: .06, range: $-.12-.15)(\mathrm{p}<.03)$ and $\mathrm{H}($ mean: .04, range: $-.07-.20 ; \mathrm{p}<.05)$ from 200 to $400 \mathrm{~ms}$.

No further significant effect was found.

\section{Predictive estimates of TULIA scores on frontal theta power}

The linear regression model revealed a significant main effect of the TULIA test

$(\mathrm{F}(12,5)=3.2, \mathrm{p}<0.05, \mathrm{r} 2=.72, \mathrm{r} 2$ adjusted $=.67)$ over a fronto-central cluster of electrodes

380 (FC1, C1). More specifically, we found a significant direct relation $(\beta=.85, \mathrm{p}<.01)$ between 381 theta power and TULIA scores in the time-range from 200 to $400 \mathrm{~ms}$ (Figure 6, panel A). No 
other main effect nor interaction were found for the other predictors (i.e., brain lesion volume, days post stroke, FAB scores, and words comprehension; Figure 6, panel B) within the same time-window at that electrode site.

Descriptive statistics shows that $\mathrm{S} 2$ were the most prevalent errors $(\mathrm{M}=12 ; \mathrm{SD}=4.69)$, followed by $\mathrm{S} 0(\mathrm{M}=4.83 ; \mathrm{SD}=3.25), \mathrm{S} 1(\mathrm{M}=4.33 ; \mathrm{SD}=1.03)$ and $\mathrm{S} 3(\mathrm{M}=1 ; \mathrm{SD}=9.89) . \mathrm{S} 2$ errors refer to a difficulty of apraxic patients to correct the trajectory of a gesture, and committing errors without correction. S0 errors refer to severe problems in executing the movement, and S1 index problems in both trajectory and semantic content of the movement. S3 errors (the least frequent) include the correction of ongoing movements.

\section{Tract disconnection probability}

Tract disconnection probability (mean, standard deviation, and number of patients for each group that showed greater than 0.5 probability of disconnection) for both LA+ and LAare shown in Table 6. T-test comparison with false-discovery rate correction for multiple comparisons did not show significant differences between groups

\section{Subjective reports of virtual embodiment}

Table 3 reports average ownership and vicarious agency ratings in LA+, LA- and healthy controls. Individual ratings were entered in a mixed-design ANOVA with GROUP (LA+, LA-, H) as between-subjects factor, and EMBODIMENT (2 levels: Ow vs. Ag), ACCURACY (2 levels: C vs. I) and LIMB (2 levels: R vs. L) as within-subjects factors. Newman-Keuls post-hoc test was adopted for multiple comparisons. The ANOVA resulted in a significant main effect of the ACCURACY $\left(\mathrm{F}_{1,19}=7.6, \mathrm{p}<0.02, \eta^{2}=0.28\right)$, explained by overall higher values of Embodiment for Correct (mean $\pm \mathrm{SD}=0.61 \pm 0.25$ ) with respect to 
Incorrect (mean $\pm \mathrm{SD}=0.56 \pm 0.25$ ) actions. No further significant main effect nor interaction were found (all $\mathrm{ps}>0.15$ ). Moreover, subjective scores of embodiment did not correlate with any of the error-related EEG signals, namely: oPe amplitude and theta-band activity (for Ow: $\mathrm{LA}+=$ all $p s>0.2, \mathrm{LA}-=$ all $p s>0.05, \mathrm{H}=$ all $s>0.07$; for Ag: $\mathrm{LA}+=$ all $p s>0.5, \mathrm{LA}-=$ all $\mathrm{p}>0.1, \mathrm{H}=$ all $\mathrm{p} s>0.07)$.

\section{Discussion}

We explored in left brain-damaged people with or without apraxia, and in a control group of healthy individuals $(\mathrm{H})$ the electrocortical dynamics of error observation by combining immersive virtual reality and EEG recording. Results in the time and time-frequency domain showed that observation of erroneous actions brought a suppression of early oPe and theta activity in LA+ and LA-. In addition, LA+ showed a significant difference when compared to $\mathrm{H}$, that was not showed when $\mathrm{H}$ were compared to LA-, suggesting an impairment in error processing for $\mathrm{LA}+$. In addition, LA+ highlighted aberrant theta phase synchronicity between fronto-frontal and fronto-parietal networks, respect to both LA- and H. To the best of our knowledge, this study reports the first evidence of altered performance monitoring in patients with LA. Based on the theoretical framework of the conflict monitoring theories (Yeung et al., 2004; Botvinick, 2001) and of the affordance competition hypothesis (Cisek, 2007; Pezzullo and Cisek, 2016), we submit that this impairment could be driven by the LA patients' original difficulty in selecting appropriate action schema to implement goal-directed behaviours, and in suppressing inappropriate conflicting affordances arising from the observation of an object. Consequently, the excessive burden of unresolved conflict prevents patients from fluid action understanding and impairs the EEG dynamics that underpins appropriate performance monitoring. 
431 evidence in support of our hypothesis. Early Pe is a P300-like positive-going component that

432 differentiates from late Pe (Falkenstein et al., 2000) for maximally peaking over mid-frontal 433 electrodes in error trials (Ullsperger et al., 2014), and for originating from mid-frontal cortical 434 sources (Boxtel et al., 2005). Also, early Pe dissociates from the late Pe in terms of functional 435 significance. In keeping with P300 event-related brain potential theories (Polich, 2007), early 436 Pe seems resembling the activity of a task-related, frontal cognitive control mechanism 437 associated to automatic error processing (prediction errors or mismatch), whereas late Pe may 438 be linked to higher-order processes, like memory processing or affective reactions to 439 maladaptive/infrequent stimuli or internal model updating and potential adjustments (di 440 Gregorio et al., 2018; Falkenstein et al., 2000). In the present study, LA+ did not show the 441 classical early Pe following incorrect trials; LA- did not show a difference between incorrect 442 or correct actions. However, one can qualitatively appreciate how LA- showed a modulation 443 in the time series of the ERP, that is not visible in the LA+; also, when contrasts between 444 groups are performed, $\mathrm{H}$ showed a significant difference as compared to LA+, but not when 445 compared to LA-. This suggests a reduced responsivity of LA+ performance monitoring 446 system that interferes with the resolution of the conflict generated from the competition 447 between incorrect action outcomes and correct action schema (Yeung et al., 2004; Botvinick, 448 2001). Interestingly, studies demonstrate that $\mathrm{P} 300$-like waveforms originate from phasic 449 activity of the norepinephrine system and may underlie the learning processes responsible for 450 subsequent motor improvement (Nieuwenhuis et al., 2005; Yu and Dayan, 2005; Dayan and 451 Yu, 2006). Therefore, the absence of early Pe in LA+, may not only index a defective conflict 452 processing, but also an impaired ability to implement flexible behavioural adaptation in a 453 cascade-like sequence of neurocognitive events. Another relevant result of our study is the 454 absence of the observation error-related negativity (oERN) across all the subjects and 455 experimental groups. Previous studies using virtual-reality (Spinelli et al., 2018; Pezzetta et 
al, 2018; Pavone et al., 2016) or other methods (van Schie et al., 2004; Bates et al., 2005;

457 Koban et al., 2010; de Brujn and von Rehin, 2012), reported that observation of others' action

458 errors evoked an oERN in the onlookers' brain. Here, oERN suppression can be explained in

459 terms of an age-dependent effect (e.g., Gehring \& Knight, 2000; Nieuwenhuis et al., 2001;

460 Mathewson et al., 2005), or in view of the novel evidence that errors can elicit error-positivity

461 in the absence of an ERN (Di Gregorio et al., 2018; Pezzetta et al., 2021). While our results fit

462 adequately with the above options, drawing firm conclusions is likely complicated by the

463 original aim of this study and the characteristics of the sample. Absence of oERN was

464 admittedly unexpected; therefore, future works should tackle this important issue using ad-

465 hoc developed experimental designs.

466 Analyses of brain oscillatory activity provide another important support for altered

467 performance monitoring in apraxia. Indeed, our results indicate a significant error-related

468 suppression of mid-frontal theta power in the group of LA+. Cognitive control over goal-

469 directed behaviour is a highly flexible process that integrates information coming from the

470 actual context and specific task-related demands (Helfrich and Knight, 2016). A large-scale

471 network governed by the pre-frontal cortex and composed by distant and yet functionally

472 related cortical and subcortical areas (Miller and Cohen, 2001), rhythmically orchestrates such

473 integration. Electrophysiology evidence demonstrates that activity in the pre-frontal cortex

474 becomes significantly higher when deviant outcomes (Dürschmid et al., 2016) or errors

475 (Fonken et al., 2016) are detected. EEG studies in non-human primates also demonstrate that

476 this multiplexed computational activity is carried out in distinct frequency bands, time and

477 brain (scalp) locations (Akam and Kullmann, 2014). Notably, in humans, an increase of mid-

478 frontal theta power underlies error execution (Trujillo and Allen, 2007; Hanslmayr et al.,

479 2008; Cavanagh et al., 2009; 2012; Munneke et al., 2015) and error observation (Spinelli et

480 al., 2018; Pavone et al., 2016; Pezzetta et al, 2018). This effect has been convincingly 
associated to conflict processing and resolution (Cohen, 2014). Together with time-domain

482 results, the suppression of mid-frontal theta power in LA+ patients suggests that conflict

483 arising from the competition between correct and incorrect action schema is not adequately

484 resolved in the patients' performance monitoring system. Moreover, connectivity analyses

485 show a decreased theta synchronicity between fronto-frontal and fronto-parieto-occipital areas

486 in LA+ with respect to both LA- and H. Phase synchronicity reflects a coherent burst of

487 activity of neuronal populations in distant cortical regions. Such an alignment of brain

488 oscillatory dynamics in time facilitates the communication between networks and ultimately

489 enables efficient cognitive processing (Voloh et al., 2015, Daitch et al., 2013). Tellingly,

490 fronto-frontal and frontal-parietal network dynamics has been suggested to play a crucial role

491 in making fluid cognitive control (Nàcher et al., 2013; Philips et al., 2013; Gregoriou et al.,

492 2009). EEG studies show that post-error theta phase enhancement in these networks underlies

493 perceptually integration of maladaptive information, and represents a call to increase

494 cognitive control for subsequent behavioral adjustment (Cohen et al., 2009; Cavanagh et al.,

495 2009; Cohen and Cavanagh, 2011). That LA+ patients exhibit aberrant oscillatory patterns

496 during action monitoring, suggests not only a reduced capacity of their performance

497 monitoring system to resolve the conflict, but also a difficulty to capitalize on perceptual and

498 sensorimotor information flow from action observation. This latter claim fits with previous

499 reports showing that motor skills of apraxic patients may influence their visual action

500 understanding, and vice versa (Pazzaglia et al., 2008a).

501 It should be noticed that we found no difference between correct and incorrect actions in

$502 \mathrm{LA}+$ and LA- in terms of theta and Pe signals absolute values. However, further contrasts

503 between groups, obtained from incorrect minus correct actions, showed a significant

504 difference between LA+ and $\mathrm{H}$, but not between LA- and $\mathrm{H}$, thus highlighting how $\mathrm{H}$ showed

505 increased theta activity in response to errors, that was instead not found in LA+. The lack of a 
direct difference when comparing LA+ and LA- might be due to lack of sensitivity to pick up

507 differences between patients' groups due to the reduced sample. Tellingly, however,

508 connectivity analyses in the theta range show that LA+ had lower theta as compared to both

509 LA- and $\mathrm{H}$ both in the frontal and parietal regions, suggesting an impaired error-monitoring

510 process in $\mathrm{LA}+$. Another result that deserves discussion concerns the extent to which altered

511 performance monitoring parallels the apraxic phenotypes. This was tested by means of a

512 multiple linear regression model, predicting theta power activity from an index of the apraxic

513 impairment (TULIA scores) and two other main factors that significantly differed between

514 LA+ and LA-, i.e., lesioned brain volume and an index of general functionality of frontal

515 lobes (FAB scores). Results show evidence for a direct relation between the severity of

516 apraxia and error-related mid-frontal theta power, so that reduced error-related mid-frontal

517 theta power was predicted by the severity of the disease (indexed by lower TULIA scores).

518 This effect hints at the close link between the apraxic phenotype and the integrity of the

519 performance monitoring system and confirms our hypothesis that symptoms of apraxia

520 prevent patients' ability to resolve the conflict generated by the observation of incorrect

521 actions, regardless of the amount of lesioned cortical volume and of the patients' impairment

522 in frontal executive functions, as indexed by FAB scores. The data on the lesion maps

523 suggests that lesions to inferior frontal gyrus, rolandic operculum, insula, and putamen, as

524 well as to superior fronto-occipital and superior longitudinal fasciculi seem to differentiate the

525 two groups. These patterns of results are in line with previous findings showing how LA+

526 exhibit behavioral deficits during prediction, gesture comprehension and error detection tasks

527 (e.g. Kilner, 2011; Keysers and Gazzola, 2014; Avenanti, Candidi, Urgesi, 2013; Urgesi,

528 Candidi, Avenanti, 2014). Moreover, the most significant difference between the two groups

529 is represented by the involvement of the basal ganglia (i.e. putamen) and the insula in LA+ vs

530 LA-. Crucially, these regions have been found to play a role in in error detection and

531 performance monitoring (Klein et al., 2007; Falkenstein et al., 2001; Yang, Andric, Mathew, 
2015). Importantly, the superior fronto-occipital fasciculus and superior longitudinal

fasciculus were also lesioned in the LA+ group, thus supporting the hypothesis that deficits in our apraxic patients might have been due to the association between fronto-temporal, frontoparietal and basal ganglia lesions.

A final point of discussion concerns the analysis of subjective reports. In keeping with previous studies (Padrao et al., 2016; Pavone et al., 2016) embodiment scores were lower during observation of erroneous with respect to correct actions. However, here we did not find any relation between error-related EEG signatures and subjective reports of embodiment, neither in healthy $(\mathrm{H})$ nor in brain-damaged individuals (LA+ and LA-). One possible explanation may be due to having collected ratings of embodiment (ownership and vicarious agency) only in the $25 \%$ of trials which, combined with the small sample size may have determined this lack of sensitivity. Alternatively, and in keeping with previous report (Spinelli et al., 2018), one may note that the relation between virtual embodiment and error-related brain signatures is merely correlative and not causative. Future work is needed to understand whether inducing embodiment of artificial (virtual) upper limbs might play any specific role in improving the action monitoring capacity in people suffering from higher-order motor disorders. The issue of patients' sample size deserves discussion. Indeed, LA+ group and LA- count a relatively small number of individuals. This is mainly due to the adoption of very restrictive inclusion criteria based on socio-demographic data, brain-injury site, and individuals' compliance to our EEG protocol in virtual reality. Therefore, while on the one hand the selection criteria reduced the sample size, on the other it prevented us from recruiting a non-homogeneous patients' sample and jumping to misleading conclusions. However, future studies with larger cohorts of patients are recommended to replicate these results. Furthermore, we maintained the unbalance of frequency of occurrence typical of error studies by including 48 incorrect trials and 72 correct ones. Previous methodological studies 
557 have showed that increasing the number of trials does not affect the reliability of error 558 signatures and a minimum of 8 trials may be sufficient to reliably elicit ERN and Pe (Olvet \&

559 Hajcak, 2009; Pontifex et al., 2010). In conclusion, our results indicate reduced electrocortical

560 activity of the performance monitoring systems in brain damaged patients with LA+

561 suggesting that ideomotor limb apraxia brings about difficulties in error processing when

562 observing the actions of others. Our paradigm paves the way to potentially interesting new

563 studies on the role that theta-band oscillatory entrainment over pre-frontal cortices may play

564 in facilitating patients' performance monitoring. Moreover, our study casts fresh light on the

565 neuro-cognitive architecture characterizing apraxia and thus has the potential to inspire novel

566 rehabilitation protocols. 
567

568

569

570

571

572

573

574

575

576

577

578

579

580

581

582

583

584

585

586

587

588

589

590

591

592

593

\section{References}

Aglioti, S.M., Pazzaglia, M. (2010) Representing actions through their sound. Exp Brain Res. 206(2):141-51.

Aglioti, S.M., Pazzaglia, M. (2011) Sounds and scents in (social) action. Trends Cogn Sci. 5(2):47-55.

Akam, T., \& Kullmann, D. M. (2014). Oscillatory multiplexing of population codes for selective communication in the mammalian brain. Nature Reviews Neuroscience, 15(2), 111.

Angela, J. Y., \& Dayan, P. (2005). Uncertainty, neuromodulation, and attention. Neuron, 46(4), 681-692.

Appollonio, I., Leone, M., Isella, V., Piamarta, F., Consoli, T., Villa, M. L., ... \& Nichelli, P. (2005). The Frontal Assessment Battery (FAB): normative values in an Italian population sample. Neurological Sciences, $26(2), 108-116$.

Avenanti, A., Candidi, M., \& Urgesi, C. (2013). Vicarious motor activation during action perception: beyond correlational evidence. Frontiers in human neuroscience, 7, 185.

Bates, A. T., Patel, T. P., \& Liddle, P. F. (2005). External Behavior Monitoring Mirrors Internal Behavior Monitoring. Journal of Psychophysiology, 19(4), 281-288. http://doi.org/10.1027/0269-8803.19.4.281

Bizzozero, I., Costato, D., Sala, S. D., Papagno, C., Spinnler, H., \& Venneri, A. (2000). Upper and lower face apraxia: role of the right hemisphere. Brain, 123(11), 2213-2230.

Boldt, A., \& Yeung, N. (2015). Shared neural markers of decision confidence and error detection. Journal of Neuroscience, 35(8), 3478-3484.

Botvinick, M. M., Braver, T. S., Barch, D. M., Carter, C. S., \& Cohen, J. D. (2001). Conflict monitoring and cognitive control. Psychological review, 108(3), 624.

Buxbaum, L. J., \& Randerath, J. (2018). Limb apraxia and the left parietal lobe. Handbook of clinical neurology, 151, 349-363.

Buxbaum, L. J., Johnson-Frey, S. H., \& Bartlett-Williams, M. (2005). Deficient internal models for planning hand-object interactions in apraxia. Neuropsychologia, 43(6), 917-929.

Buxbaum, L. J., Shapiro, A. D., \& Coslett, H. B. (2014). Critical brain regions for tool-related and imitative actions: a componential analysis. Brain, 137(7), 1971-1985.

Buxbaum, L. J., Shapiro, A. D., \& Coslett, H. B. (2014). Reply: Apraxia: a gestural or a cognitive disorder?. Brain, 138(3), e334-e334. 

neuroscience, 12(11), 1793-1802.

Canzano, L., Scandola, M., Pernigo, S., Aglioti, S. M., \& Moro, V. (2014). Anosognosia for apraxia:

598 Experimental evidence for defective awareness of one's own bucco-facial gestures. Cortex, 61, 148-157.

Casile, A., \& Giese, M. A. (2004). Possible influences of motor learning on perception of biological motion. Journal of Vision, 4(8), 221-221. cognitive sciences, 18(8), 414-421.

Cavanagh, J. F., Cohen, M. X., \& Allen, J. J. (2009). Prelude to and resolution of an error: EEG phase synchrony reveals cognitive control dynamics during action monitoring. Journal of Neuroscience, 29(1), 98-105.

Cavanagh, J. F., Frank, M. J., Klein, T. J., \& Allen, J. J. (2010). Frontal theta links prediction errors to behavioral adaptation in reinforcement learning. Neuroimage, 49(4), 3198-3209.

Cavanagh, J. F., Zambrano-Vazquez, L., \& Allen, J. J. (2012). Theta lingua franca: A common mid-frontal substrate for action monitoring processes. Psychophysiology, 49(2), 220-238.

Cisek, P. (2007). Cortical mechanisms of action selection: the affordance competition hypothesis.

Cisek, P., \& Kalaska, J. F. (2010). Neural mechanisms for interacting with a world full of action choices. neurosciences, 37(9), 480-490.

Cohen, M. X., \& Cavanagh, J. F. (2011). Single-trial regression elucidates the role of prefrontal theta oscillations in response conflict. Frontiers in psychology, 2, 30. 
Cooper, R. P. (2007). Tool use and related errors in ideational apraxia: The quantitative simulation of patient error profiles. Cortex, 43(3), 319-337.

Cross, E. S., Kraemer, D. J., Hamilton, A. F. D. C., Kelley, W. M., \& Grafton, S. T. (2008). Sensitivity of the action observation network to physical and observational learning. Cerebral cortex, 19(2), 315-326.

Cubelli, R., Marchetti, C., Boscolo, G., \& Della Sala, S. (2000). Cognition in action: Testing a model of limb apraxia. Brain and cognition, 44(2), 144-165.

Daitch, A. L., Sharma, M., Roland, J. L., Astafiev, S. V., Bundy, D. T., Gaona, C. M., ... \& Corbetta, M. (2013). Frequency-specific mechanism links human brain networks for spatial attention. Proceedings of the National Academy of Sciences, 110(48), 19585-19590.

Dayan, P., \& Yu, A. J. (2006). Phasic norepinephrine: a neural interrupt signal for unexpected events. Network: Computation in Neural Systems, 17(4), 335-350.

De Bruijn, E. R., \& von Rhein, D. T. (2012). Is your error my concern? An event-related potential study on own and observed error detection in cooperation and competition. Frontiers in neuroscience, $6,8$.

de Bruijn, E. R., Schubotz, R. I., \& Ullsperger, M. (2007). An event-related potential study on the observation of erroneous everyday actions. Cognitive, Affective, \& Behavioral Neuroscience, 7(4), 278-285.

de Haan, B., \& Karnath, H. O. (2018). A hitchhiker's guide to lesion-behaviour mapping. Neuropsychologia, 115, 5-16.

\section{De Renzi, E., \& Lucchelli, F. (1988). Ideational apraxia. Brain, 111(5), 1173-1185}

Di Gregorio, F., Maier, M. E., \& Steinhauser, M. (2018). Errors can elicit an error positivity in the absence of an error negativity: Evidence for independent systems of human error monitoring. NeuroImage.

Dürschmid, S., Edwards, E., Reichert, C., Dewar, C., Hinrichs, H., Heinze, H. J., ... \& Knight, R. T. (2016). Hierarchy of prediction errors for auditory events in human temporal and frontal cortex. Proceedings of the National Academy of Sciences, 113(24), 6755-6760.

Endrass, T., Reuter, B., \& Kathmann, N. (2007). ERP correlates of conscious error recognition: aware and unaware errors in an antisaccade task. European Journal of Neuroscience, 26(6), 1714-1720.

Ernst, B., \& Steinhauser, M. (2017). Top-down control over feedback processing: The probability of valid feedback affects feedback-related brain activity. Brain and cognition, 115, 33-40. 
648

649

650

651

652

653

654

655

656

657

658

659

660

661

662

663

664

665

666

667

668

669

670

671

672

673

674

675

Ertelt, D., Small, S., Solodkin, A., Dettmers, C., McNamara, A., Binkofski, F., \& Buccino, G. (2007). Action observation has a positive impact on rehabilitation of motor deficits after stroke. Neuroimage, 36, T164-T173.

Falkenstein, M., Hoormann, J., Christ, S., \& Hohnsbein, J. (2000). ERP components on reaction errors and their functional significance: a tutorial. Biological psychology, 51(2-3), 87-107.

Falkenstein, M., Hielscher, H., Dziobek, I., Schwarzenau, P., Hoormann, J., Sundermann, B., \& Hohnsbein, J. (2001). Action monitoring, error detection, and the basal ganglia: an ERP study. Neuroreport, 12(1), 157-161.

Fonken, Y. M., Rieger, J. W., Tzvi, E., Crone, N. E., Chang, E., Parvizi, J., ... \& Krämer, U. M. (2016). Frontal and motor cortex contributions to response inhibition: evidence from electrocorticography. Journal of neurophysiology, 115(4), 2224-2236.

Foulon, C., Cerliani, L., Kinkingnehun, S., Levy, R., Rosso, C., Urbanski, M., ... \& Thiebaut de Schotten, M. (2018). Advanced lesion symptom mapping analyses and implementation as BCBtoolkit. Gigascience, 7(3), giy004.

Gehring, W. J., \& Knight, R. T. (2000). Prefrontal-cingulate interactions in action monitoring. Nature neuroscience, 3(5), 516.

Gillies, M., \& Spanlang, B. (2010). Comparing and evaluating real time character engines for virtual environments. Presence: Teleoperators and Virtual Environments, 19(2), 95-117.

Gregoriou, G. G., Gotts, S. J., Zhou, H., \& Desimone, R. (2009). High-frequency, long-range coupling between prefrontal and visual cortex during attention. science, 324(5931), 1207-1210.

Halsband, U., Schmitt, J., Weyers, M., Binkofski, F., Grützner, G., \& Freund, H. J. (2001). Recognition and imitation of pantomimed motor acts after unilateral parietal and premotor lesions: A perspective on apraxia. Neuropsychologia, 39(2), 200-216.

Hanslmayr, S., Pastötter, B., Bäuml, K. H., Gruber, S., Wimber, M., \& Klimesch, W. (2008). The electrophysiological dynamics of interference during the Stroop task. Journal of Cognitive Neuroscience, 20(2), 215-225.

Helfrich, R. F., \& Knight, R. T. (2016). Oscillatory dynamics of prefrontal cognitive control. Trends in cognitive sciences, 20(12), 916-930.

Jax, S. A., \& Buxbaum, L. J. (2013). Response interference between functional and structural object-related actions is increased in patients with ideomotor apraxia. Journal of neuropsychology, 7(1), 12-18. 
676

677

678

679

680

681

682

683

684

685

686

687

688

Karnath, H.-O., \& Rennig, J. (2016). Investigating structure and function in the healthy human brain: validity of acute versus chronic lesion-symptom mapping. Brain Structure and Function, 222(5), 20592070.doi:10.1007/s00429-016-1325-7

Keysers, C., \& Gazzola, V. (2014). Hebbian learning and predictive mirror neurons for actions, sensations and emotions. Phil. Trans. R. Soc. B, 369(1644), 20130175.

Kilner, J. M. (2011). More than one pathway to action understanding. Trends in cognitive sciences, 15(8), 352357.

Klein, T. A., Endrass, T., Kathmann, N., Neumann, J., von Cramon, D. Y., \& Ullsperger, M. (2007). Neural correlates of error awareness. Neuroimage, 34(4), 1774-1781.

Koban, L., Pourtois, G., Vocat, R., \& Vuilleumier, P. (2010). When your errors make me lose or win: eventrelated potentials to observed errors of cooperators and competitors. Social Neuroscience, 5(4), 360-374.

Lachaux, J. P., Rodriguez, E., Martinerie, J., \& Varela, F. J. (1999). Measuring phase synchrony in brain signals. Human brain mapping, 8(4), 194-208.

Lepage, J. F., \& Théoret, H. (2006). EEG evidence for the presence of an action observation-execution matching system in children. European Journal of Neuroscience, 23(9), 2505-2510.

Luzzatti, C., Willmes, K., \& De Bleser, R. (1996). Aachener aphasie test: versione italiana. Firenze: Organizzazioni Speciali.

Maris, E., \& Oostenveld, R. (2007). Nonparametric statistical testing of EEG-and MEG-data. Journal of neuroscience methods, 164(1), 177-190.

Mathewson, K. J., Dywan, J., \& Segalowitz, S. J. (2005). Brain bases of error-related ERPs as influenced by age and task. Biological psychology, 70(2), 88-104.

Miller, E. K., \& Cohen, J. D. (2001). An integrative theory of prefrontal cortex function. Annual review of neuroscience, 24(1), 167-202.

Munneke, G. J., Nap, T. S., Schippers, E. E., \& Cohen, M. X. (2015). A statistical comparison of EEG timeand time-frequency domain representations of error processing. Brain research, 1618, 222-230.

Nácher, V., Ledberg, A., Deco, G., \& Romo, R. (2013). Coherent delta-band oscillations between cortical areas correlate with decision making. Proceedings of the National Academy of Sciences, 110(37), 15085-15090.

Nieuwenhuis, S., Aston-Jones, G., \& Cohen, J. D. (2005). Decision making, the P3, and the locus coeruleus-norepinephrine system. Psychological bulletin, 131(4), 510. 

are differentially related to awareness of response errors: Evidence from an antisaccade task. Psychophysiology, $38(5), 752-760$

Olvet, D. M., \& Hajcak, G. (2009). The stability of error-related brain activity with increasing trials. Psychophysiology, 46(5), 957-961.

Oostenveld, R., Fries, P., Maris, E., \& Schoffelen, J. M. (2011). FieldTrip: open source software for advanced analysis of MEG, EEG, and invasive electrophysiological data. Computational intelligence and neuroscience, 2011,1 .

Pavone, E. F., Tieri, G., Rizza, G., Tidoni, E., Grisoni, L., \& Aglioti, S. M. (2016). Embodying others in immersive virtual reality: electro-cortical signatures of monitoring the errors in the actions of an avatar seen from a first-person perspective. Journal of Neuroscience, 36(2), 268-279.

Pazzaglia, M., Pizzamiglio, L., Pes, E., \& Aglioti, S. M. (2008b). The sound of actions in apraxia. Current biology, 18(22), 1766-1772.

Pazzaglia, M., Smania, N., Corato, E., \& Aglioti, S. M. (2008a). Neural underpinnings of gesture discrimination in patients with limb apraxia. Journal of Neuroscience, 28(12), 3030-3041.

Pezzetta, R., Nicolardi, V., Tidoni, E., \& Aglioti, S. M. (2018). Error, rather than its probability, elicits specific electrocortical signatures: a combined EEG-immersive virtual reality study of action observation. Journal of neurophysiology.

R. Pezzetta, M. Wokke, S.M. Aglioti, R. Ridderinkhof, Doing it wrong: a systematic review on electrocortical and behavioral correlates of error monitoring in patients with neurological disorders, Neuroscience (2021), doi: https://doi.org/10.1016/j.neuroscience.2021.01.027

Pezzulo, G., \& Cisek, P. (2016). Navigating the affordance landscape: feedback control as a process model of behavior and cognition. Trends in cognitive sciences, 20(6), 414-424.

Pontifex, M. B., Scudder, M. R., Brown, M. L., O'Leary, K. C., Wu, C. T., Themanson, J. R., \& Hillman, C.

729 H. (2010). On the number of trials necessary for stabilization of error-related brain activity across the life 730 span. Psychophysiology, 47(4), 767-773.

Phillips, J. M., Vinck, M., Everling, S., \& Womelsdorf, T. (2013). A long-range fronto-parietal 5-to 10-Hz 732 network predicts “top-down” controlled guidance in a task-switch paradigm. Cerebral Cortex, 24(8), 1996-2008. 
Raven, J. C. Court JH, Raven J (1988) Manual for Raven's progressive matrices and vocabulary scales.

Ridderinkhof, K. R., Ramautar, J. R., \& Wijnen, J. G. (2009). To PE or not to PE: A P3-like ERP component the frontal lobe connections and their variability

Rothi, L. J., Heilman, K. M., \& Watson, R. T. (1985). Pantomime comprehension and ideomotor apraxia.

Journal of Neurology, Neurosurgery \& Psychiatry, 48(3), 207-210.

Rounis, E., \& Humphreys, G. (2015). Limb apraxia and the "affordance competition hypothesis". Frontiers in Human Neuroscience, 9, 429.

Sauseng, P., \& Klimesch, W. (2008). What does phase information of oscillatory brain activity tell us about cognitive processes?. Neuroscience \& Biobehavioral Reviews, 32(5), 1001-1013.

Scandola, M., Canzano, L., Avesani, R., Leder, M., Bertagnoli, S., Gobbetto, V., ... \& Moro, V. (2021). Anosognosia for limb and bucco-facial apraxia as inferred from the recognition of gestural errors. Journal of neuropsychology, 15(1), 20-45.

Sirigu, A., Daprati, E., Pradat-Diehl, P., Franck, N., \& Jeannerod, M. (1999). Perception of self-generated movement following left parietal lesion. Brain, 122(10), 1867-1874.

Spinelli, G., Tieri, G., Pavone, E. F., \& Aglioti, S. M. (2018). Wronger than wrong: graded mapping of the errors of an avatar in the performance monitoring system of the onlooker. NeuroImage, 167, 1-10.

756 Steinhauser, M., \& Yeung, N. (2010). Decision processes in human performance monitoring. Journal of Neuroscience, 30(46), 15643-15653. flexible framework for wide-spectrum VR development. Presence: Teleoperators and Virtual Environments, 19(4), 302-312. 
Thiebaut de Schotten, M., Tomaiuolo, F., Aiello, M., Merola, S., Silvetti, M., Lecce, F., ... \& Doricchi, F. (2014). Damage to white matter pathways in subacute and chronic spatial neglect: a group study and 2 singlecase studies with complete virtual "in vivo" tractography dissection. Cerebral cortex, 24(3), 691-706.

Trujillo, L. T., \& Allen, J. J. (2007). Theta EEG dynamics of the error-related negativity. Clinical Neurophysiology, 118(3), 645-668.

Ullsperger, M., Fischer, A. G., Nigbur, R., \& Endrass, T. (2014). Neural mechanisms and temporal dynamics of performance monitoring. Trends in Cognitive Sciences, 18(5), 259-267.

Urgesi, C., Candidi, M., \& Avenanti, A. (2014). Neuroanatomical substrates of action perception and understanding: an anatomic likelihood estimation meta-analysis of lesion-symptom mapping studies in brain injured patients. Frontiers in human neuroscience, $8,344$.

Van Boxtel, G. J., Van Der Molen, M. W., \& Jennings, J. R. (2005). Differential involvement of the anterior cingulate cortex in performance monitoring during a stop-signal task. Journal of Psychophysiology, 19(1), 1.

van Driel, J., Ridderinkhof, K. R., \& Cohen, M. X. (2012). Not all errors are alike: theta and alpha EEG dynamics relate to differences in error-processing dynamics. Journal of Neuroscience, 32(47), 16795-16806.

van Schie, H. T., Mars, R. B., Coles, M. G., \& Bekkering, H. (2004). Modulation of activity in medial frontal and motor cortices during error observation. Nature neuroscience, 7(5), 549.

Vanbellingen, T., Kersten, B., Van Hemelrijk, B., Van de Winckel, A., Bertschi, M., Müri, R., ... \& Bohlhalter, S. (2010). Comprehensive assessment of gesture production: a new test of upper limb apraxia (TULIA). European journal of neurology, 17(1), 59-66

Vinck, M., Oostenveld, R., Van Wingerden, M., Battaglia, F., \& Pennartz, C. M. (2011). An improved index of phase-synchronization for electrophysiological data in the presence of volume-conduction, noise and samplesize bias. Neuroimage, 55(4), 1548-1565.

Voloh, B., Valiante, T. A., Everling, S., \& Womelsdorf, T. (2015). Theta-gamma coordination between anterior cingulate and prefrontal cortex indexes correct attention shifts. Proceedings of the National Academy of Sciences, 112(27), 8457-8462.

Watson, C. E., \& Buxbaum, L. J. (2015). A distributed network critical for selecting among tool-directed actions. Cortex, 65, 65-82. 
Wilson, B., Cockburn, J., \& Halligan, P. (1987). Development of a behavioral test of visuospatial neglect. Archives of physical medicine and rehabilitation, 68(2), 98-102.

Yang, J., Andric, M., \& Mathew, M. M. (2015). The neural basis of hand gesture comprehension: a metaanalysis of functional magnetic resonance imaging sudies. Neuroscience \& Biobehavioral Reviews, 57, 88-104. Sep;15(9):1307-12. doi: 10.1038/nn.3180. Epub 2012 Aug 5.

Figures legends

Figure 1 - Brain lesion analysis. Colour rendering of the lesion patterns in LA+ (A panel) and LA(B panel) patients. The $\mathrm{C}$ panel represents the LA+ minus LA- subtraction. The figure shows the typical pattern of fronto-parietal damage typical of apraxia.

Figure 2 - Apparatus and experimental task. Panel A: a four-screens CAVE system (left) and a snapshot of an actual experimental trial (right) depicting a participant seeing a virtual limb from 1PP during the EEG recording. Panel B: render of the virtual scenario as seen from the 1PP. The avatar has its own upper limbs placed on the table at $\sim 50 \mathrm{~cm}$ from the virtual glass (left). On the right-side, combinations of avatar's action outcomes that participants observed in the four experimental conditions (ACCURACY [Correct, Incorrect] * LIMB [Right, Left]).

Figure 3 - ERPs analysis. Panel A: topographical maps of the early oPe in the time range from 300 to

$809700 \mathrm{~ms}$, for each group (LA+, LA- and H) and each condition (correct and incorrect), and for the difference incorrect minus correct condition. Panel B: time-course of early oPe for each group (LA+,

811 LA- and H) in correct (red) and incorrect (blue) condition at the significant fronto-central cluster of electrodes (i.e., FC1, FCz, FC2, C1). The gray box highlights significant time-points at which early

813 oPe voltage differs between incorrect vs. correct condition. Right-ward topographical maps show the significant fronto-central cluster (white markers) resulting from the contrast between incorrect minus 
correct condition, for each group (LA+, LA- and $\mathrm{H}$ ) in the time-range from 430 to $550 \mathrm{~ms}$. Panel C:

816 time-course of early oPe (upper-row) for each group (LA+, LA- and H) at the mid-frontal cluster. The gray box highlights significant time-points in which early oPe voltage differs between groups ( $\mathrm{H}$ vs

818 LA+, H vs. LA- and LA- vs LA+). Lower-row shows significant mid-frontal and parieto-occipital 819 clusters (white markers) resulting from the contrast between groups (H vs LA+, H vs. LA- and LA- vs $\mathrm{LA}+$ ) in the time range from 420 to $560 \mathrm{~ms}$.

821 Figure 4 - Time-frequency analysis. Panel A: theta band-power differences (black contour-line) resulting from the contrast between incorrect and correct condition for each group (LA+, LA- and H) along the whole frequency spectrum (from 4 to $30 \mathrm{~Hz}$ ). Right-ward topographical maps depict cluster of electrodes (white markers) in which theta band-power activity differ between incorrect vs correct condition (time-window from 300 to $650 \mathrm{~ms}$ ). Panel B: upper-row shows statistical differences of theta band-power activity resulting from the contrast between groups ( $\mathrm{H}$ vs. LA+, $\mathrm{H}$ vs. LA- and LAvs. LA+). The bottom row depicts significant clusters of electrodes in which theta band-power activity (4-8 Hz) differ between groups (white asterisks).

Figure 5 - Phase connectivity analysis. Theta phase connectivity between mid-frontal (FC1, FCz, $\mathrm{FC} 2, \mathrm{C} 1, \mathrm{Cz}, \mathrm{C} 2)$, parieto-occipital (PO7, PO3, POz, PO4, PO8, 01, 0z, O2) and lateral pre-frontal electrodes (F6, F4, F3, F5), for each group (LA+, LA- and H). Values refer to the difference between incorrect and correct condition and are plotted from 4 to $15 \mathrm{~Hz}$ for visualization purposes. Topographical maps depict theta connectivity between mid-frontal (white markers), lateral pre-frontal (red diamonds) and parieto-occipital electrodes (violet diamonds) in three time-windows (200-400, 400-600 and 600-800 ms), for each group (LA+, LA- and H).

Figure 6 - Figure 6 - Link between apraxic phenotypes and mid-frontal theta oscillations. Multiple Linear Regression between TULIA scores, brain lesioned volume, FAB scores, days post

838 stroke, word comprehension, and power spectra. Panel A shows the main effect of TULIA scores: leftcolumn displays $\beta$ coefficients in the time-frequency space over the significant cluster of electrodes 840 (FC1/C1); right-column depicts the relation between theta-band power and TULIA scores in the timewindow running from 200 to $400 \mathrm{~ms}$. Black contour-line highlights the time-window and the 
842 frequencies at which the effect is significant. Panel B shows the non-significant effect of brain lesion

843 volume (left-column), years post stroke (central-left column), FAB scores (central right-column) and

844 words comprehension (right-column) on theta-band power in the time windows from 200 to $400 \mathrm{~ms}$.

845

846

847

848

849

850 Tables

851 Table 1 - Demographic and clinical data

\begin{tabular}{|c|c|c|c|c|c|c|c|c|c|c|}
\hline Participant & $\begin{array}{c}\text { Age } \\
\text { (years) }\end{array}$ & $\begin{array}{l}\text { Education } \\
\text { (years) }\end{array}$ & $\begin{array}{c}\text { Interval } \\
\text { from lesion } \\
\text { (days) }\end{array}$ & $\begin{array}{l}\text { Raven } \\
(10 \mathrm{~min})\end{array}$ & TULIA & $\begin{array}{l}\text { Apraxia of } \\
\text { utilization }\end{array}$ & $\begin{array}{c}\text { Word } \\
\text { Comprehension }\end{array}$ & $\begin{array}{c}\text { Sentence } \\
\text { Comprehension }\end{array}$ & $\begin{array}{c}\text { FAB } \\
\text { tot 3-6 } \\
\text { (mean) }\end{array}$ & $\begin{array}{c}\text { Line } \\
\text { Bisection }\end{array}$ \\
\hline LA-1 & 70 & 13 & 563 & 32.5 & 222 & 14 & 30 & 28 & 3 & 9 \\
\hline LA-2 & 41 & 18 & 531 & 30 & 228 & 14 & 28 & 30 & 2.7 & 9 \\
\hline LA-3 & 63 & 13 & 627 & 29.5 & 231 & 14 & 30 & 24 & 3 & 9 \\
\hline LA-4 & 39 & 13 & 619 & 27 & 228 & 14 & 29 & 26 & 3 & 8 \\
\hline LA-5 & 51 & 13 & 688 & 32 & 234 & 14 & 30 & 30 & 3 & 9 \\
\hline LA-6 & 80 & 13 & 1095 & 26 & 228 & 14 & 30 & 22 & 3 & 9 \\
\hline Mean & 57.33 & 13.83 & 687.17 & 28.75 & 228.5 & 14 & 29.5 & 26.67 & 3 & 8.83 \\
\hline$( \pm \mathrm{SD})$ & $(16.43)$ & (2.04) & $(207.08)$ & $(2.75)$ & (3.99) & $(0)$ & $(0.83)$ & $(3.27)$ & (0) & $(0.41)$ \\
\hline $\mathrm{LA}+1$ & 70 & 8 & 473 & 16.5 & 127 & 12 & 20 & 25 & 2 & 9 \\
\hline $\mathrm{LA}+2$ & 80 & 13 & 532 & 16.5 & 137 & 14 & 28 & 19 & 1 & 7 \\
\hline $\mathrm{LA}+3$ & 68 & 17 & 498 & 31.5 & 180 & 14 & 27 & 13 & 2 & 8 \\
\hline $\mathrm{LA}+4$ & 33 & 13 & 648 & 31.5 & 165 & 14 & 26 & 26 & 3 & 9 \\
\hline $\mathrm{LA}+5$ & 78 & 8 & 292 & 24.5 & 162 & 14 & 23 & 17 & 2 & 8 \\
\hline $\mathrm{LA}+6$ & 68 & 13 & 1039 & 24.5 & 192 & 14 & 30 & 25 & 2 & 9 \\
\hline Mean & 66.17 & 12 & 580.33 & 24. 17 & 160.5 & 13.67 & 25.67 & 20.83 & 2 & 8.33 \\
\hline$( \pm \mathrm{SD})$ & $(17.05)$ & $(3.46)$ & $(252.48)$ & (6.69) & $(24.78)$ & $(0.82)$ & (3.61) & $(5.31)$ & $(0.63)$ & $(0.82)$ \\
\hline Z-score & -0.800 & 0.880 & -2.081 & 1.601 & 2.882 & 0.48 & 2.161 & 1.841 & 2.321 & 1.04 \\
\hline p-value & 0.423 & 0.378 & $0.037^{*}$ & 0.109 & $0.003 *$ & 0.630 & $0.03 *$ & 0.06 & $0.02 *$ & 0.297 \\
\hline
\end{tabular}

852 All patients are in their chronic stage according to Karnath \& Rennig (2016). Asterisks indicate significance

853 between groups (Mann-Whitney U test).

854

855

856 
Table 2 - Trials count after artifact-rejection

\begin{tabular}{ccccc}
\hline & \multicolumn{2}{c}{ Right } & \multicolumn{2}{c}{ Left } \\
\hline & $\begin{array}{c}\text { correct } \\
\text { (out of 36) }\end{array}$ & $\begin{array}{c}\text { incorrect } \\
\text { (out of 24) }\end{array}$ & $\begin{array}{c}\text { correct } \\
\text { (out of 36) }\end{array}$ & $\begin{array}{c}\text { incorrect } \\
\text { (out of 24) }\end{array}$ \\
\hline LA+ & $33.0(92 \%) ;$ & $23.0(96 \%) ;$ & $34.3(95 \%) ;$ & $23.3(97 \%) ;$ \\
(mean; \%; range) & $32-34$ & $22-24$ & $33-36$ & $20-26$ \\
LA- & $35.0(97 \%) ;$ & $23.6(97 \%) ;$ & $34.9(97 \%) ;$ & $23.5(97 \%) ;$ \\
(mean; \%; range) & $33-36$ & $22-24$ & $33-36$ & $22-24$ \\
H & $34.0(94 \%) ;$ & $22.8(95 \%) ;$ & $34.0(94 \%) ;$ & $22.7(95 \%) ;$ \\
(mean; \%; range) & $28-38$ & $18-27$ & $28-38$ & $18-27$ \\
\hline
\end{tabular}

Table 3 - Subjective ratings of Ownership and Agency.

\begin{tabular}{|c|c|c|c|c|c|c|c|c|}
\hline & \multicolumn{4}{|c|}{ Ownership } & \multicolumn{4}{|c|}{ Vicarious Agency } \\
\hline & \multicolumn{2}{|c|}{ Right } & \multicolumn{2}{|c|}{ Left } & \multicolumn{2}{|c|}{ Right } & \multicolumn{2}{|c|}{ Left } \\
\hline & Correct & Incorrect & Correct & Incorrect & Correct & Incorrect & Correct & Incorrect \\
\hline $\mathrm{LA}+$ & $.57 \pm .40$ & $.58 \pm .38$ & $.60 \pm .40$ & $.61 \pm .39$ & $.58 \pm .38$ & $.58 \pm .36$ & $.59 \pm .41$ & $.58 \pm .41$ \\
\hline LA- & $.44 \pm .28$ & $.41 \pm .28$ & $.48 \pm .29$ & $.38 \pm .31$ & $.45 \pm .28$ & $.43 \pm .28$ & $.48 \pm .30$ & $.38 \pm .32$ \\
\hline $\mathrm{H}$ & $.37 \pm .23$ & $.29 \pm .20$ & $.35 \pm .21$ & $.27 \pm .20$ & $.36 \pm .24$ & $.25 \pm .15$ & $.33 \pm .22$ & $.25 \pm .18$ \\
\hline
\end{tabular}




\begin{tabular}{|c|c|c|c|c|c|}
\hline $\mathrm{LA}+$ & & & & & \\
\hline Area & $\begin{array}{c}\text { Number of lesioned } \\
\text { voxels }\end{array}$ & $\begin{array}{c}\% \text { of lesioned } \\
\text { voxels }\end{array}$ & $\operatorname{MaxX}$ & MaxY & MaxZ \\
\hline Frontal_Inf_Oper_L & 1169 & 14 & -36 & 5 & 23 \\
\hline Frontal_Inf_Tri_L & 2048 & 10 & -40 & 21 & -1 \\
\hline Rolandic_Oper_L & 3453 & 43 & -45 & -10 & 22 \\
\hline Insula_L & 8349 & 55 & -39 & -9 & 24 \\
\hline Putamen_L & 1348 & 17 & -31 & 10 & -1 \\
\hline Heschl_L & 103 & 6 & -47 & -11 & 3 \\
\hline Anterior_limb_of_int & 541 & 17 & -26 & 7 & 17 \\
\hline Anterior_corona_rad & 3228 & 47 & -28 & 11 & 20 \\
\hline Posterior_corona_rad & 750 & 20 & -30 & -31 & 26 \\
\hline Superior_corona_rad & 4647 & 62 & -29 & -2 & 19 \\
\hline External_capsule_R & 2146 & 38 & -32 & 9 & -1 \\
\hline Superior_longitudina & 2936 & 44 & -33 & -3 & 21 \\
\hline Superior_fronto-occi & 356 & 70 & -24 & 4 & 19 \\
\hline \multicolumn{6}{|l|}{ LA- } \\
\hline Area & $\begin{array}{c}\text { Number of lesioned } \\
\text { voxels }\end{array}$ & $\begin{array}{c}\% \text { of lesioned } \\
\text { voxels }\end{array}$ & $\operatorname{MaxX}$ & $\operatorname{MaxY}$ & MaxZ \\
\hline Rolandic_Oper_L & 452 & 6 & -46 & -1 & 6 \\
\hline Postcentral_L & 2108 & 7 & -66 & -14 & 14 \\
\hline SupraMarginal_L & 1710 & 17 & -67 & -26 & 26 \\
\hline
\end{tabular}

Table 5 - LA+ and LA- subtraction lesion map

\begin{tabular}{|c|c|c|c|c|c|}
\hline \multicolumn{6}{|c|}{ Subtraction 6 LA+ minus 6 LA- (lesioned voxels in at least 3 patients) } \\
\hline Area & $\begin{array}{l}\begin{array}{l}\text { Number } \\
\text { voxels }\end{array} \\
\text { of lesioned }\end{array}$ & $\%$ of lesioned voxels & $\operatorname{MaxX}$ & MaxY & MaxZ \\
\hline Frontal_Inf_Oper_L & 786 & 9 & -36 & 5 & 23 \\
\hline Frontal_Inf_Tri_L & 2025 & 10 & -40 & 21 & -1 \\
\hline Rolandic_Oper_L & 1636 & 21 & -42 & -2 & 12 \\
\hline Insula_L & 7392 & 49 & -37 & -9 & 24 \\
\hline Putamen_L & 1348 & 17 & -31 & 10 & -1 \\
\hline Anterior_limb_of_int & 541 & 17 & -26 & 7 & 17 \\
\hline Anterior_corona_radi & 2976 & 43 & -28 & 11 & 20 \\
\hline Superior_corona_radi & 4267 & 57 & -29 & -2 & 19 \\
\hline Posterior_corona_rad & 750 & 20 & -30 & -31 & 26 \\
\hline External_capsule_R & 2146 & 38 & -32 & 9 & -1 \\
\hline Superior_longitudina & 2784 & 42 & -33 & -3 & 21 \\
\hline Superior fronto-occi & 351 & 69 & -24 & 4 & 19 \\
\hline
\end{tabular}




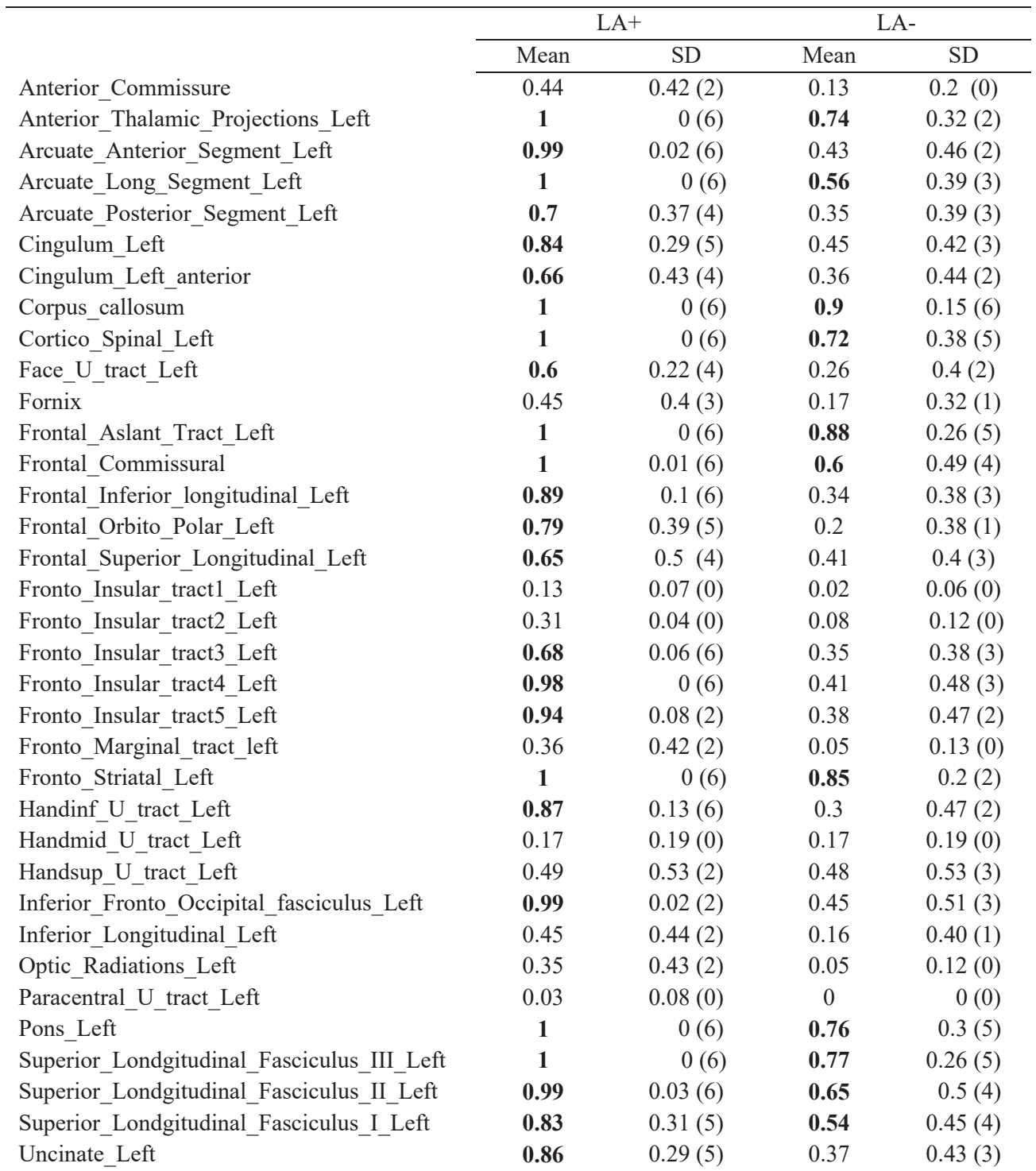

For a given lesion, Tractotron provides a probability of disconnection for almost all known tracts (Foulon et al.

875 2018). The probability corresponds to the lesioned voxel with the highest \% value; therefore, patients with a

876 probability of disconnection $>50 \%(=0.5)$ are usually considered as disconnected. Values of 1 indicate maximal

877 probability of tract disconnection. Tracts that exceed the $50 \%$ of probability of disconnection are shown in bold.

878 The table shows for each tract the mean value, the standard deviation and the number of patients that exceed the

8790.5 probability of disconnection, for each group. 
$\mathrm{LA}+$

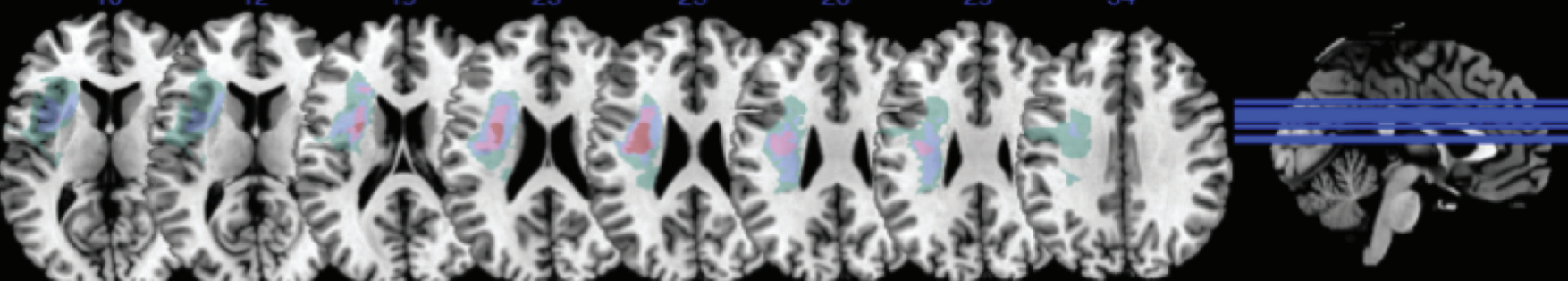

(1) E (2)

U

2

$$
\text { L R }
$$

$\mathrm{N}$ of lesioned patients

B

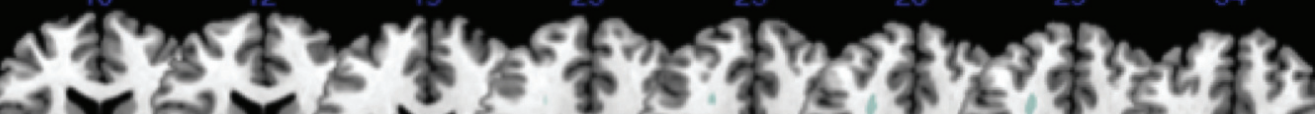
造, ह

L R

(a)

는

(2)

(a)

()

())

$\varangle$

(2)

낭

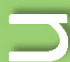

(a)

$Z$

(a)

술

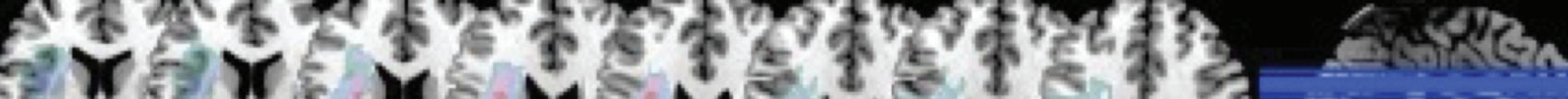

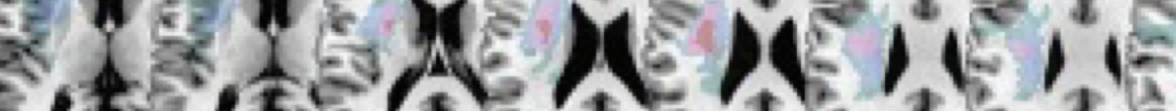

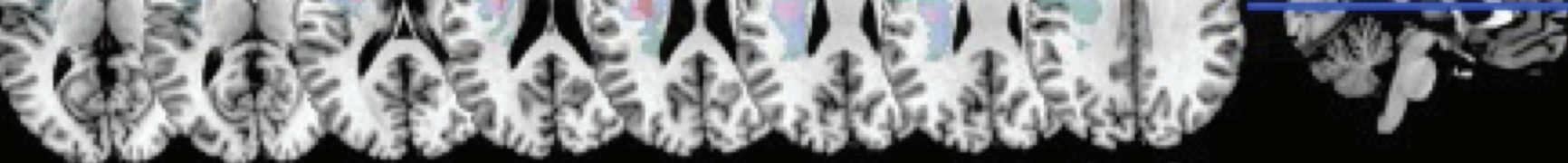

\section{$\mathrm{N}$ of lesioned patients}



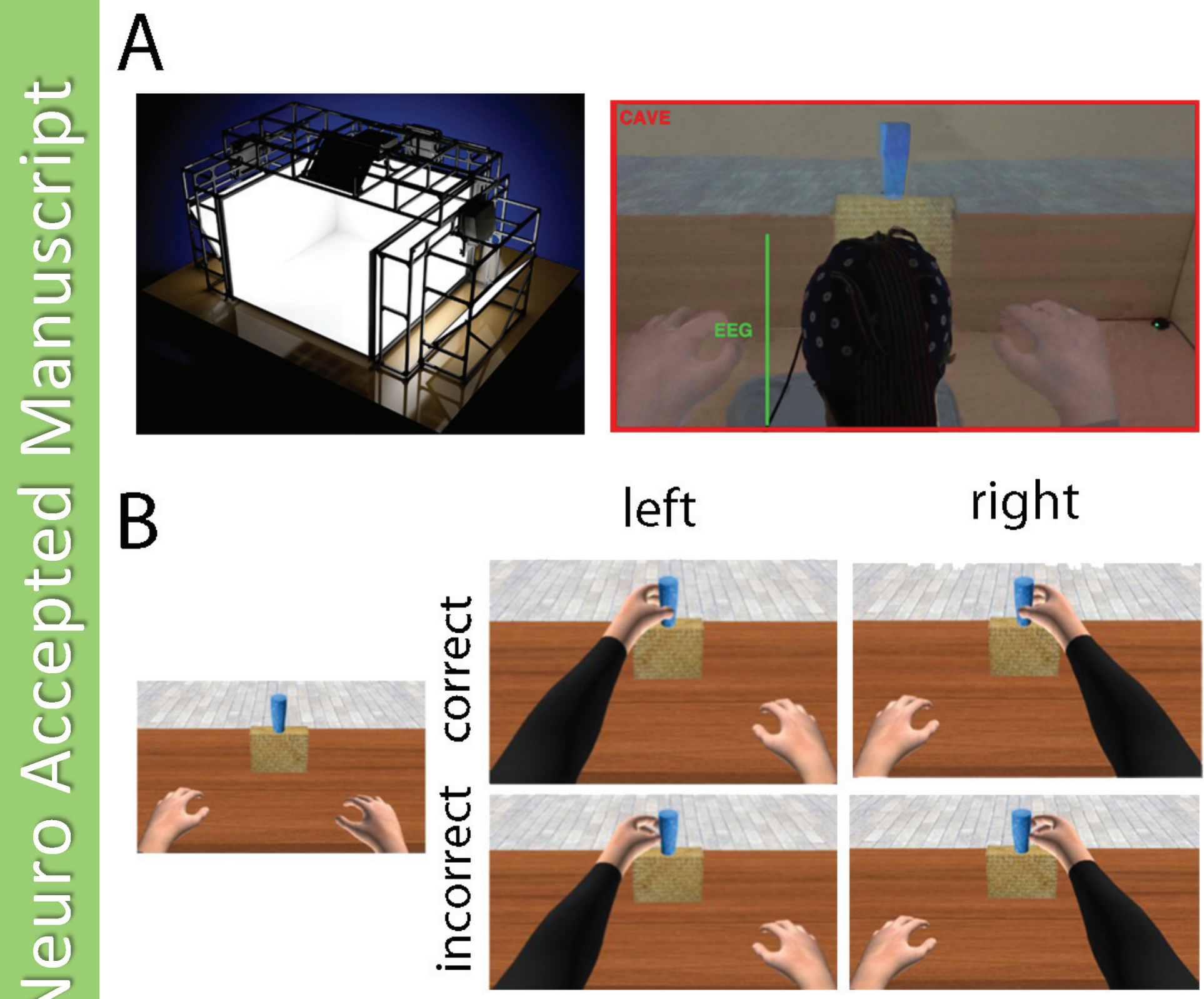
A

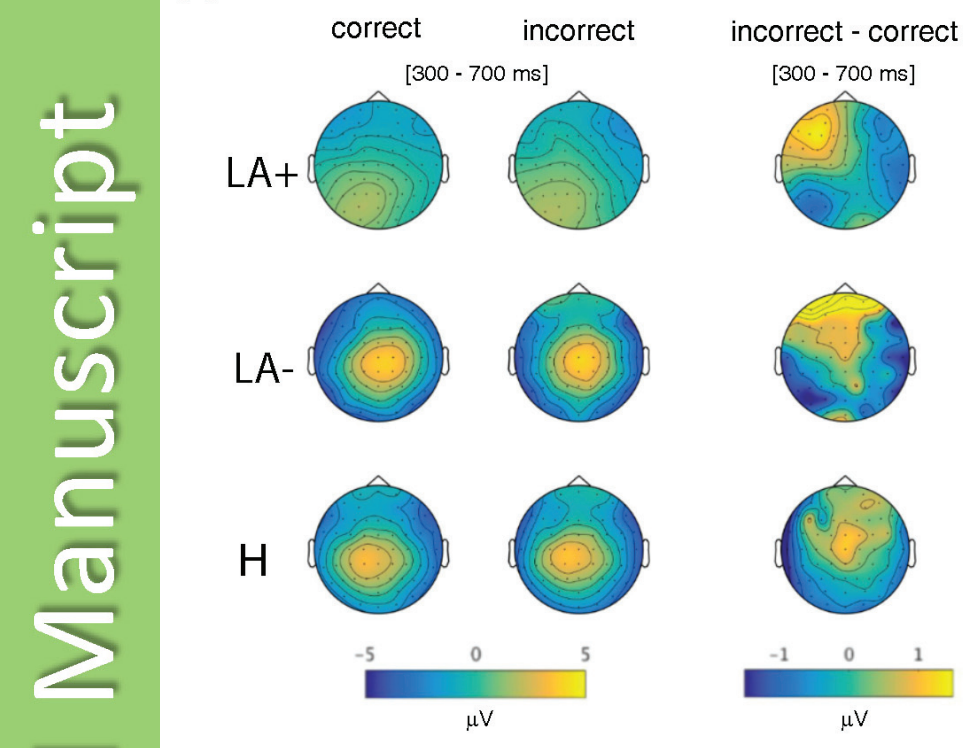

B

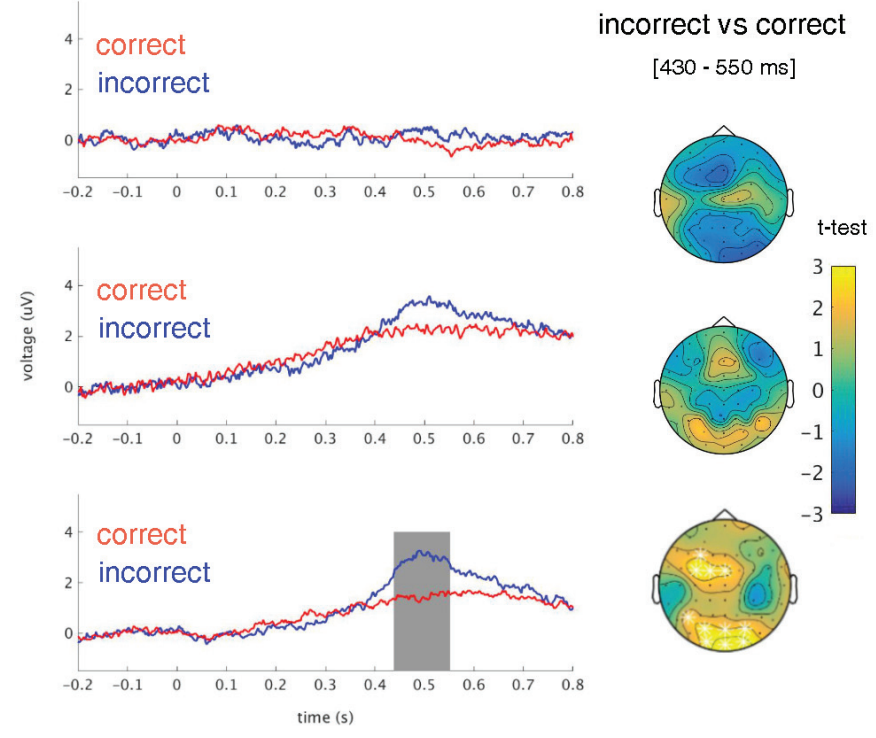

(d) $\mathrm{c}$

ㄴ)

@

(i)

(u)

(u)

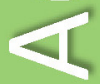

(2)

늑

(a)

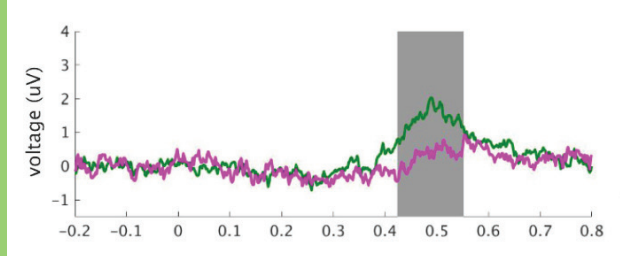

H vs. LA+

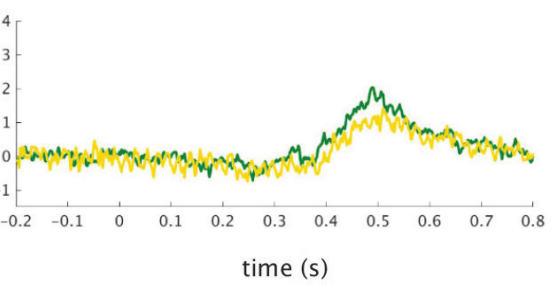

H vs. LA-

LA- vs. LA+

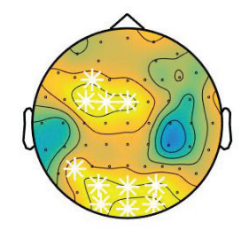

[420 - $560 \mathrm{~ms}$ ]

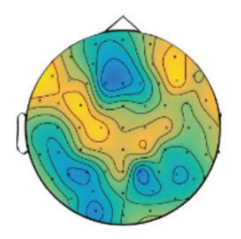

[420 - $560 \mathrm{~ms}$ ]

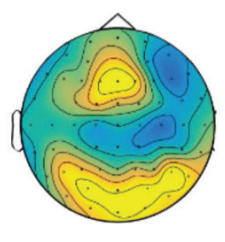

[420 - $560 \mathrm{~ms}$ ]
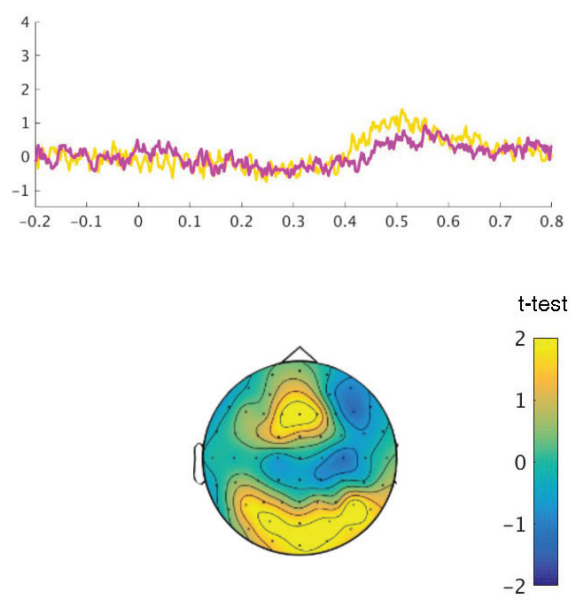


\section{A}

$\mathrm{FCz}$

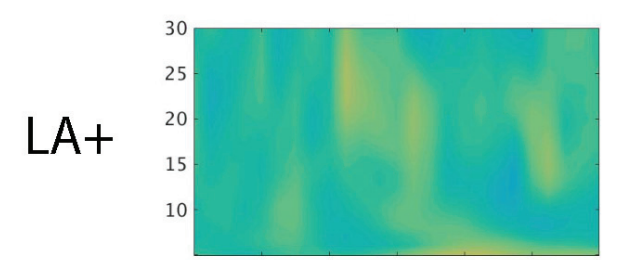

\section{incorrect vs correct}

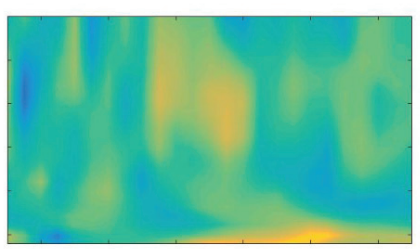

B

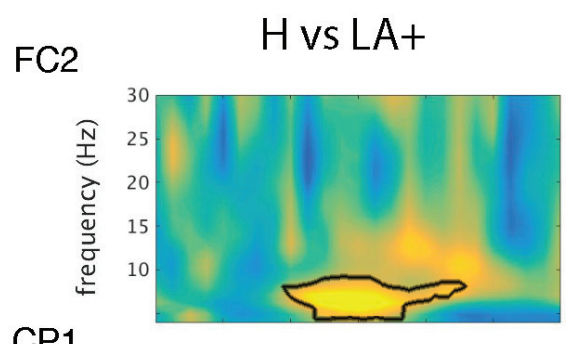

CP1
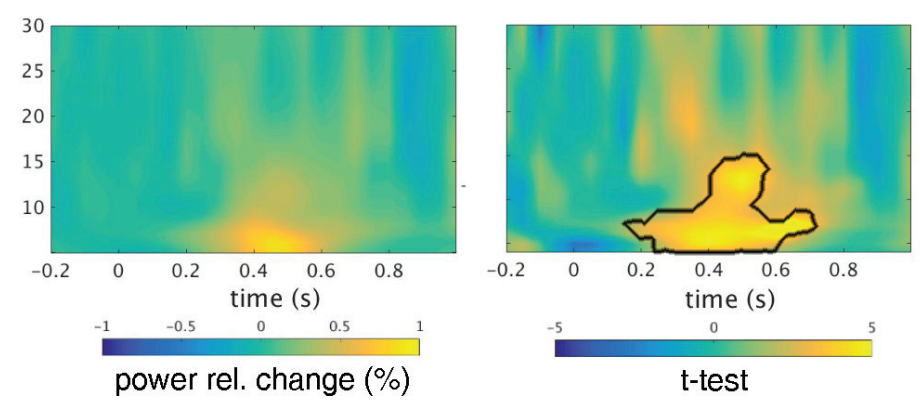

H vs LA-
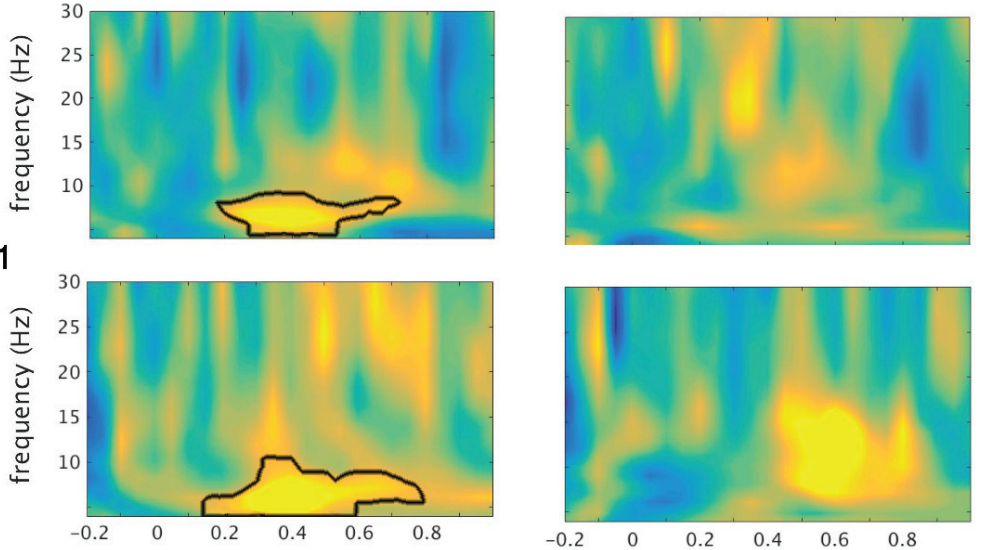

theta (4-8 Hz)

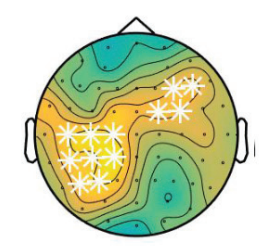

[220 - $575 \mathrm{~ms}$ ]

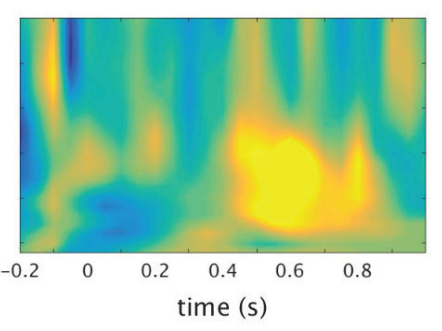

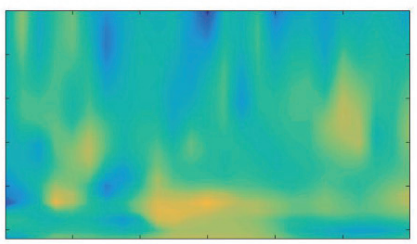
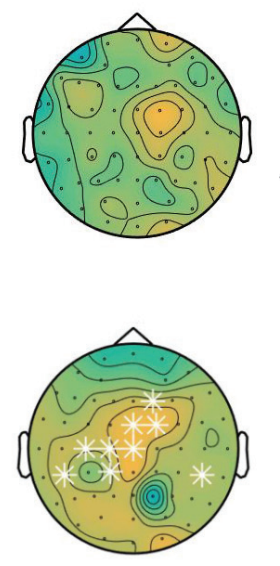

theta $(4-8 \mathrm{~Hz})$ [300 - $650 \mathrm{~ms}$ ]

LA- vs LA+

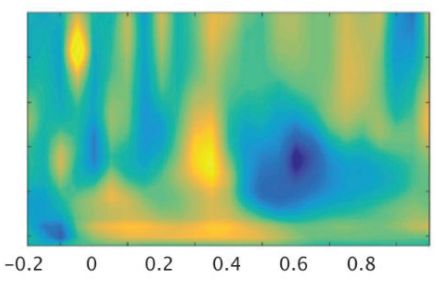

t-test

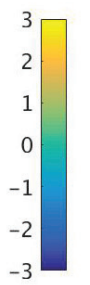

t-test
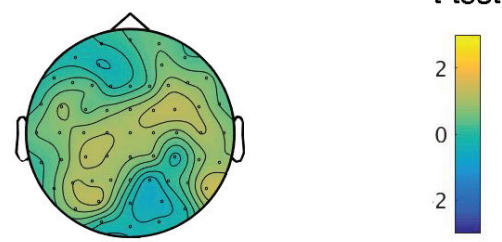

[220 - $575 \mathrm{~ms}$ ] 
incorrect - correct

mid-frontal to:

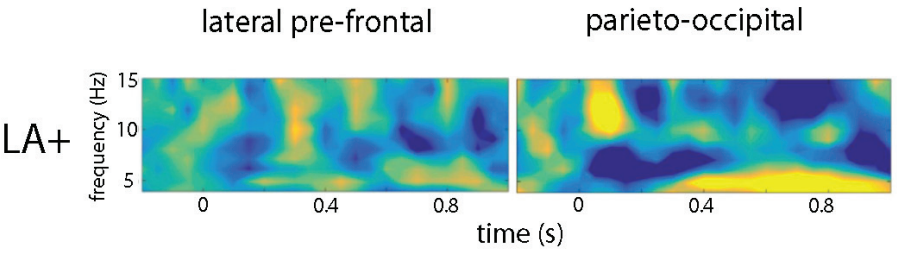

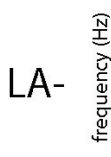

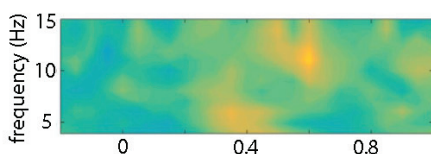

time (s)
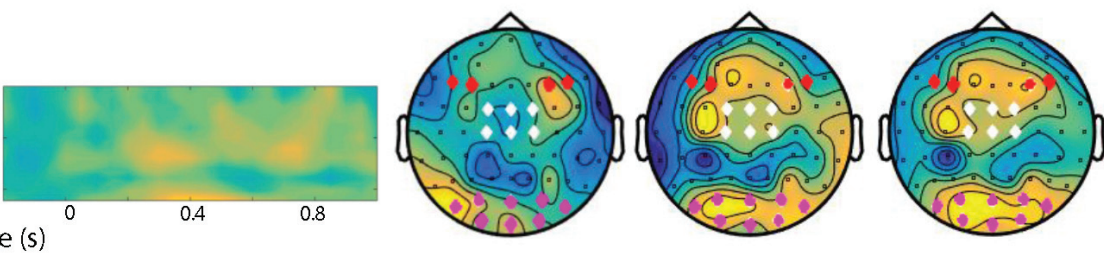

PLV

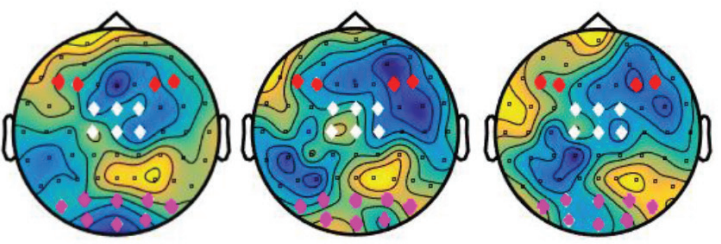

0.01
005
0
005
0.01

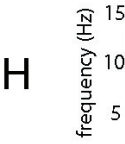
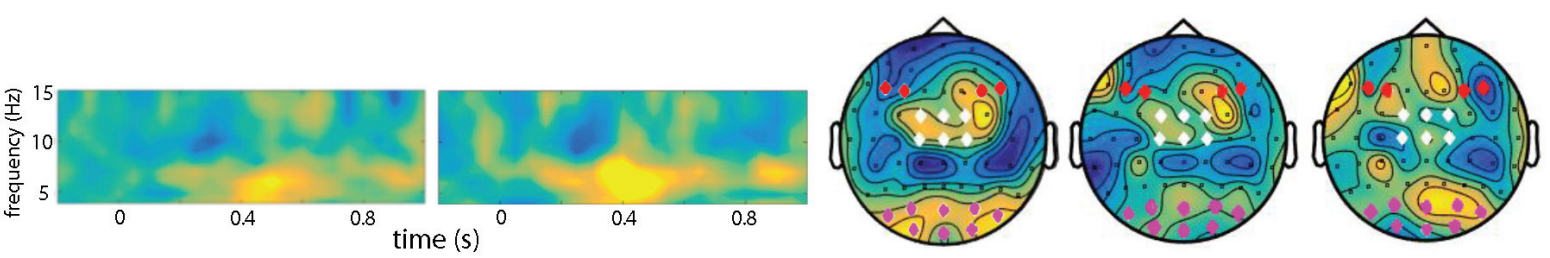

[200 - $400 \mathrm{~ms}]$
[400 - $600 \mathrm{~ms}]$

[600 - $800 \mathrm{~ms}]$ 
A

\section{TULIA scores}

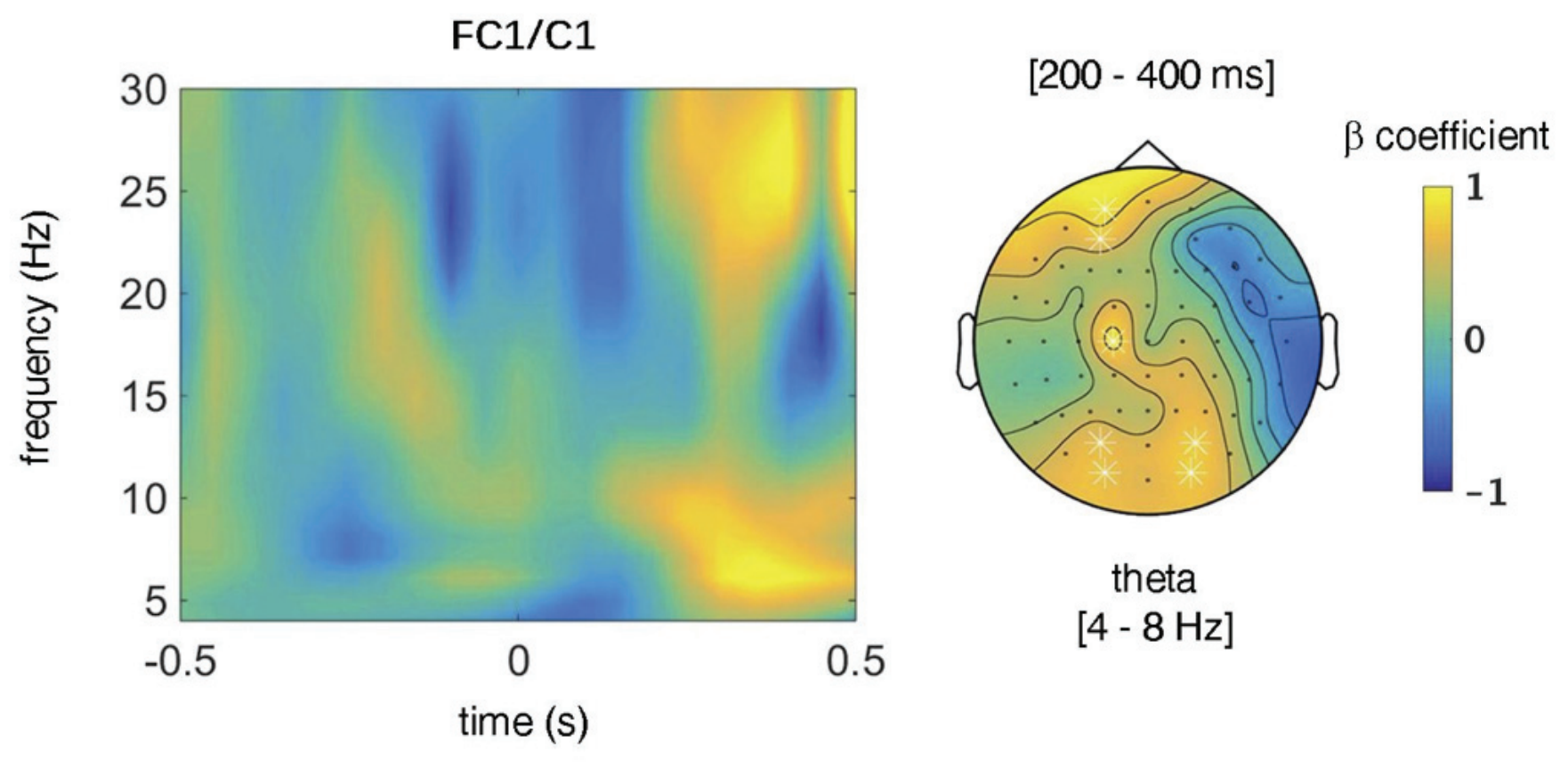

B
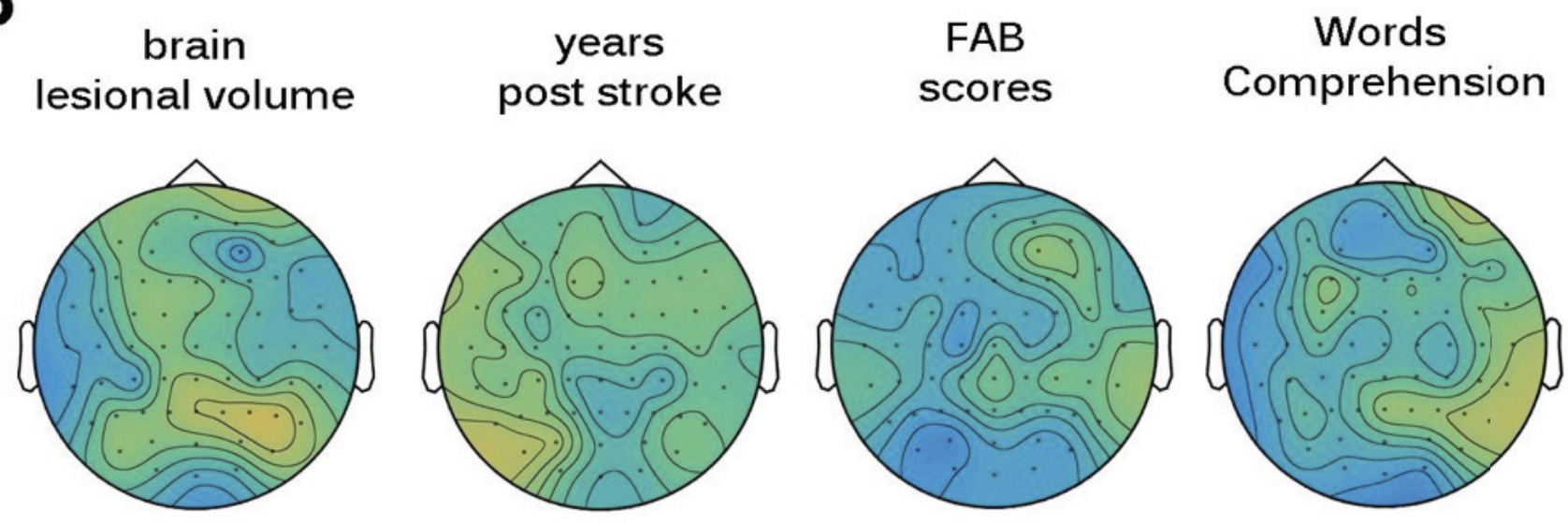

[200 - $400 \mathrm{~ms}$ ]
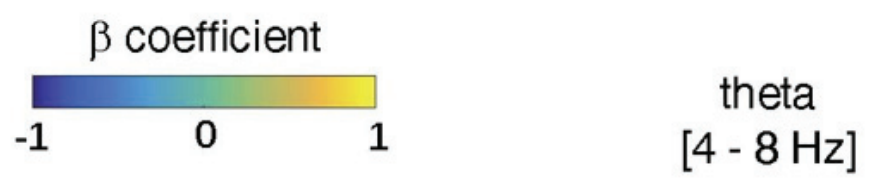